\title{
Multilane Microscopic Modeling to Measure Mobility and Safety Consequences of Mixed Traffic in Freeway Weaving Sections
}

\author{
Mudasser Seraj iD and Tony Z. Qiu (D) \\ Department of Civil and Environmental Engineering, University of Alberta, Edmonton, Alberta T6G 2R3, Canada \\ Correspondence should be addressed to Mudasser Seraj; seraj@ualberta.ca
}

Received 18 December 2020; Revised 6 May 2021; Accepted 29 June 2021; Published 15 July 2021

Academic Editor: Vittorio Astarita

Copyright (c) 2021 Mudasser Seraj and Tony Z. Qiu. This is an open access article distributed under the Creative Commons Attribution License, which permits unrestricted use, distribution, and reproduction in any medium, provided the original work is properly cited.

\begin{abstract}
Weaving sections are components of highway networks that introduce a heightened likelihood for bottlenecks and collisions. Automated vehicle technology could address this as it holds considerable promise for transportation mobility and safety improvements. However, the implications of combining automated vehicles (AuVs) with traditional human-driven vehicles (HuVs) in weaving freeway sections have not been quantitatively measured. To address this gap, this paper objectively experimented with bidirectional (i.e., longitudinal and lateral) motion dynamics in a microscopic modeling framework to measure the mobility and safety implications for mixed traffic movement in a freeway weaving section. Our research begins by establishing a multilane microscopic model for studied vehicle types (i.e., $\mathrm{AuV}$ and $\mathrm{HuV}$ ) from model predictive control with the provision to form a $\mathrm{CACC}$ platoon of $\mathrm{AuV}$ vehicles. The proposed modeling framework was tested first with $\mathrm{HuV}$ only on a two-lane weaving section and validated using standardized macroscopic parameters from the Highway Capacity Manual. This model was then applied to incrementally expand the AuV share for varying inflow rates of traffic. Simulation results showed that the maximum flow rate through the weaving section was attained at a $65 \% \mathrm{AuV}$ share. At the same time, steadiness in the average speed of traffic was experienced with increasing $\mathrm{AuV}$ share. The results also revealed that a 95\% AuV share could reduce potential conflicts by $94.28 \%$. Finally, the results of simulated scenarios were consolidated and scaled to report expected mobility and safety outcomes from the prevailing traffic state and the optimal AuV share for the current inflow rate in weaving sections.
\end{abstract}

\section{Introduction}

The rise of automated vehicle ( $\mathrm{AuV})$ technology, as an essential component of a new generation of traffic infrastructure, has been researched by academics and industry who recognize its advantages over the existing transportation framework regarding improved mobility and enhanced safety and reduced environmental impact. However, large-scale transitions to $\mathrm{AuV}$ technology-based transportation systems cannot happen overnight. Research on the fusion of such technology with the current, humanoriented transportation system that also considers the restraints of existing roadways is warranted. Certainly, freeway weaving sections are considered restraints since they act as recurrent bottleneck locations due to inherent vehicle trajectory patterns formed by vehicles changing lanes from auxiliary lanes to mainlines and vice versa. As such, substantial research has established that both mobility and safety of the weaving sections are compromised [1-8].

Although numerous studies have established the eminence of mixed traffic over traditional traffic systems from mobility, safety, and environmental perspectives, the exploration of coexistence is primarily limited to partial motion dynamics, most often car-following strategy, of studied vehicle groups [9-12]. This limitation is significant since both traffic operational and regulatory authorities must base their strategic investment and policy and legislative decision-making on sound, objective facts regarding integrating AVs with conventional traffic systems. Motivated by this gap in knowledge, this research, as part of a broader study, addresses the following question: how can the mobility and safety of varying traffic states in a multilane weaving section be influenced by the shared presence of AuVs and human-driven vehicles (HuVs)? The two-fold 
objective of this descriptive research includes the following: (i) contriving a comprehensive and realistic modeling framework of mixed traffic with bidirectional motion dynamic and (ii) quantifying and clarifying the causal connection between the presence of AuVs in traffic with a potential shift in mobility and safety benchmarks for a weaving section.

The findings of this study aim to contribute to the existing body of knowledge, which currently lacks substantial evidence to support the effect of $\mathrm{AuV}$ integration to improve the mobility and safety of weaving freeway sections. Furthermore, the study outcomes can benefit research communities and industries actively committed to intelligent transportation systems and presume that AuVs will play a significant role in overcoming flow efficiency limitations and crash likelihood. The demand for understanding the implications of $\mathrm{AuV}$ substantiates the need for more comprehensive research from the mobility and safety perspective. These perspectives play critical roles in measuring transportation system performance and the effects of mixed traffic on planning decisions. From planning and operational standpoints, traffic mobility is defined as the ability and level of ease of moving goods and services [13]. For instance, freeways can provide designated high occupancy vehicle lanes to increase the overall efficiency of moving people while maintaining the overall number of vehicles. Since a detailed evaluation of mobility shift would require a considerable mobilization of resources, a few mobility parameters are selected in this study to provide a general overview of potential adjustments to traffic mobility. On the other hand, implications on traffic safety can be measured directly from the number of collisions, injuries, and fatalities. Since AuVs are not yet widespread in present traffic, direct measures of potential changes cannot be obtained in most cases. Hence, surrogate safety indicators are adopted to measure positive or negative shifts in overall traffic safety.

\section{Literature Review}

Despite real-world pilots of AuVs and the significant advancement in knowledge on this technology, large-scale deployment of AuVs in contemporary traffic streams is not readily achievable, making much of the existing literature that studies the effects of mixed traffic reliant on traffic simulation. Model predictive control (MPC)-based microscopic traffic dynamic has been adopted in few studies to simulate AuV motion. Liu et al. [14] proposed a distributed MPC for cooperative highway driving considering safety, efficiency, and fuel economy. The cooperative lane-changing maneuver was the foundation block for the dynamics in a mixed traffic environment that recognizes the effect of surrounding $\mathrm{HuVs}$ on the cooperation procedure. Bertoni et al. [15] took an energy-saving eco-CACC approach to use nonlinear MPC to vary distance and speed between electric vehicles to minimize fuel consumption. Stanger and del Re [16] took a similar approach to demonstrate the fuel and roadway capacity benefits resulting from CACC. Kamal et al. [17] proposed an efficient driving system based on MPC where $\mathrm{AuV}$ received vehicle status of surrounding vehicles to generate optimal control decisions. Their study revealed that the proposed system improved the fuel economy and travel efficiency of the AuVs significantly.

Several simulation-based studies conducted mobility analysis for mixed traffic through capacity shifts [9, 18-27]. These studies provided valuable insights about roadway capacity changes of mainstream traffic resulting from mixed traffic flow at varying market shares, although few explicitly explores the influence of integrating AuVs into freeway weaving sections. Furthermore, the few studies that included car-following $[10,12,28]$ lack the inclusion of weaving sections and their influence on lane-changing vehicles. In addition to capacity, the impact of AuVs on mobility has been evaluated through traffic speed [29-31]. However, the aggregated impact on both of these parameters was underexplored by these studies. Malikopoulos et al. [32] examined environmental implications, travel time, and traffic throughput in mixed traffic scenarios. Rios-Torres and Malikopoulos [33] discussed the environmental and mobility aspects of mixed traffic flow for merging segments in a simulated environment. Even though weaving sections are critical components of a freeway system, scarcely any study examined the possibility of mobility and safety paradigm shifts in these sections due to introducing AuVs in the traffic stream. Fazio et al. [6] used a simulated conflict rates count to identify hazardous locations, comparing this with obtained crash rates. Uno et al. [7] identified the potential conflicts in weaving sections by analyzing vehicle movements from recorded videos. Tilg et al. [34] proposed a mixed traffic model calibrated to replicate the traffic dynamics on a weaving section. Although findings from this study revealed the potential of $\mathrm{AuVs}$ to improve the capacity of the weaving section, other aspects of mobility and safety remain uncharted. Ye and Yamamoto [35, 36] conducted studies on heterogenous traffic flow and concluded that the resulting capacity improvements depend largely on $\mathrm{AuV}$ market penetration and car-following parameters. Both studies discussed the changes in the macroscopic fundamental diagram to determine the changes in traffic flow parameters.

Several studies have been published in recent years that measure the safety impact of AuVs when mixed with human-driven vehicles (HuVs). The study conducted by Hayes [37] reported that fatality rates could be reduced to $1 \%$ of current rates once AVs reached a $100 \%$ market share. Alternatively, Fagnant and Kockelman [38] predicted that the influence of AuVs could reduce the crash rate by $90 \%$ with the elimination of human error possibility. Multiple research papers explored the simulation approach to estimate traffic safety [39-42]. Fan et al. [39] proposed a two-stage process to use the VISSIM simulation model outputs to calibrate for the surrogate safety assessment model at merging locations. A similar objective was followed by Huang et al. [40] for signalized intersections. On the other hand, Essa and Sayed [41] studied the transferability of the calibrated parameters to different sites for simulation. From the perspective of the safety impact evaluation of mixed traffic, Ye and Yamamoto [11] simulated mixed traffic to study traffic safety under various market shares of connected-automated vehicles 
(CAVs). They argued that the cautious and accurate carfollowing strategy from CAVs would significantly contribute to traffic safety. Papadoulis et al. [43] developed a bidirectional decision-making control algorithm for $\mathrm{AuVs}$ to evaluate resulting safety implications at different market penetration rates. The results revealed that traffic conflicts could be reduced by up to $90-94 \%$ with a full $\mathrm{AuV}$ traffic stream. The safety implications of exclusive AuV lanes were identified by Zhang et al. [44] with different market shares and for multiple traffic demand scenarios. This study indicated that a higher number of lanes would be required at high-demand scenarios to attain significant safety improvements. In brief, the research efforts in measuring safety implications of $\mathrm{AuVs}$ in mixed traffic environment are summarized in Table 1.

An overall review of the relevant literature solidifies the common assumption that introducing AuVs in traditional transportation systems would generate favorable mobility and safety implications. However, uncertainties regarding the magnitude of improvements as well as potential negative consequences remain equivocal. Furthermore, most studies considered collective implications from different configurations of roadway segments, which diluted the influence of natural impediment and variability resulting from individual segment structure. Hence, this research consciously identified a prevalent bottleneck segment of a freeway and explored both mobility and safety implications from a system-level prospect.

\section{A Framework of Multilane Traffic}

The framework applied in this study incorporates longitudinal and lateral motion dynamics of both vehicle types for unimpeded movement along the roadway. In addition to mandatory lane-changing, platoon formation among AuVs is also configured within this framework. More definitive conclusions can be drawn from such a comprehensive modeling architecture, directly examining the mobility and safety implications of AuVs. Agent-based modeling was adapted from the MATLAB library to develop the core model structure of this study. Agents of two different types were defined to represent the two vehicle types (i.e., $\mathrm{HuV}$ and $\mathrm{AuV}$ ). These two agents enter and exit a predefined roadway segment with average headway input to maintain average inflow rate and $\mathrm{AuV}$ share for each simulated scenario. The roadway segments were defined by giving several lanes and length of section values as inputs. The driving strategy and vehicle model were similar for both agents, apart from acceleration/deceleration conditions and platoon formation provisions. Irrespective of the leading vehicle type, $\mathrm{HuVs}$ had an average desired headway of $1.4 \mathrm{sec}$ with a standard deviation of 0.3 seconds to account for human driving behavior variability. While the desired headway of an individual vehicle would be fixed (with some exceptions for forced mandatory lane-changing) throughout the simulation period, the parameter would follow a log-normal distribution for the overall $\mathrm{HuV}$ proportion of traffic. Instead of a constant desired headway, the provision of varying desired headway distribution of $\mathrm{HuVs}$ within the modeling framework presented the opportunity to incorporate the behavioral variation of human drivers.
On the other hand, AuVs inherited desired headways from platoon configurations (i.e., interplatoon headway and intraplatoon headway) depending on their position within a platoon. As mentioned in Seraj et al. [10], the AuVs are programmed to form platoons amongst themselves. If there is an $\mathrm{HuV}$ in front of $\mathrm{AuV}$, the subject $\mathrm{AuV}$ will pursue $\mathrm{ACC}$ with a small desired headway (i.e., $1.25 \mathrm{sec}$ ) in the car-following state. However, if the leading vehicle is an $\mathrm{AuV}$, the subject $\mathrm{AuV}$ will reduce the desired headway (i.e., $1.0 \mathrm{sec}$ ) to form a CACC platoon. The maximum platoon formation length was $3 \mathrm{AuVs}$ in the simulation. Also, intraplatoon distance is taken as $4 \mathrm{sec}$ to accommodate lane-changing vehicles in between platoons. Hence, if the leading $\mathrm{AuV}$ is the $3 \mathrm{rd} \mathrm{AuV}$ in the platoon, then the subject $\mathrm{AuV}$ would maintain a 4 -sec headway.

The agent-based modelling's default driving strategy and vehicle model were customized to develop more realistic vehicle dynamics. Various modeling approaches in the literature established the spatial-temporal anticipation ability of human drivers [51-57]. The anticipative nature of human drivers compensated for higher reaction time than the ACC/CACC system that can generate quicker reactions to an event [58]. Hence, for an advanced vehicle control system, incorporating such anticipative intelligence naturally improves driving efficiency and opportunities to avoid a collision. In response, the MPC-based motion algorithm is chosen for modeling both vehicle types. MPC utilizes present information to predict the future state by controlling the process through the minimization of objective function under constraints. This study aimed at developing a control framework that combined connectivity and automation of AuVs to navigate effortlessly in mixed traffic scenarios. Our modeling framework used the MPC framework integrated into the MATLAB Stateflow model to decide between the different states of the driving strategy.

Each vehicle was assigned a vehicle ID to specify their presence on the road and vehicle type to decide on control provisions. The subject vehicle's longitudinal and lateral control was implemented by computing the current traffic state from input data and predicting its future pattern. A $\mathrm{P}$-step prediction horizon and a C-step control horizon were considered for optimizing the control inputs of the subject vehicle. The prediction and control horizon of the MPC was taken as 30 and 3 timesteps, respectively. Since a lanechanging maneuver takes multiple seconds to complete, the following constraint was included:

$$
0 \leq \delta_{r / l}(P) \leq 1,
$$

which implies that the subject vehicle was allowed to change lane only once either to the right $\left(\delta_{r}\right)$ or to the left $\left(\delta_{l}\right)$ lane within the prediction horizon $(P)$. Some additional constraints were defined to ensure safety and comfort in driving. The speed of the subject vehicle at any timestep was bounded by the following upper and lower limit:

$$
0 \leq v_{s}(k) \leq v_{\max }=1.1 \times v_{\mathrm{SL}} .
$$

Here, $v_{\max }$ is the maximum achievable speed, which is $10 \%$ higher than the speed limit, and $v_{\mathrm{SL}}=25 \mathrm{~m} / \mathrm{s}$. Acceleration is bounded by the following boundary constraints: 
TABLE 1: Research summary of safety in mixed traffic environments.

\begin{tabular}{|c|c|c|}
\hline Reference & Safety parameters & Findings \\
\hline$[45]$ & $\begin{array}{l}\text { (i) Time-to-collision (TTC); (ii) nondimensional } \\
\text { warning index }\end{array}$ & $\begin{array}{c}\text { ACC strategy can prevent vehicle gaps from reducing to an unsafe level } \\
\text { in different driving scenarios }\end{array}$ \\
\hline$[46]$ & $\begin{array}{l}\text { (i) Time Exposed Time-to-collision (TET); (ii) Time } \\
\text { Integrated Time-to-collision (TIT) }\end{array}$ & $90 \%$ reduction of rear-end collision risks due to CACC system \\
\hline$[47]$ & (i) TTC; (ii) postencroachment time (PET) & $\begin{array}{l}\text { Low CAV penetration showed an increase in potential conflicts at lower } \\
\text { headway and signalized intersections; higher CAV market share showed } \\
\text { a global decrease }\end{array}$ \\
\hline$[48]$ & (i) TET; (ii) TIT & $\begin{array}{c}\text { Degradation from CACC to ACC had a significantly negative impact on } \\
\text { longitudinal safety }\end{array}$ \\
\hline$[43]$ & (i) TTC; (ii) PET & $\begin{array}{l}\text { Estimated conflict rates reduced with a gradual increase in market share } \\
\text { of CAV and finally reduced by } 90-94 \% \text { at } 100 \% \text { CAV penetration rate }\end{array}$ \\
\hline$[49]$ & (i) TTC; (ii) TET; (iii) TIT & $\begin{array}{c}\text { Mixed traffic flow can induce higher longitudinal collision risk at low } \\
\text { market penetration of ACC driven vehicle due to individual } \\
\text { heterogeneity }\end{array}$ \\
\hline$[50]$ & (i) Number of conflicts; (ii) longitudinal volatility & $\begin{array}{c}\text { Substantial safety improvement was observed at market penetration } \\
\text { higher than } 40 \%\end{array}$ \\
\hline
\end{tabular}

$$
-3 \mathrm{~m} / \mathrm{s}^{2}=a_{\min } \leq a_{s}(k) \leq a_{\max }=2 \mathrm{~m} / \mathrm{s}^{2} .
$$

Previous studies on modeling $\mathrm{HuV}$ and $\mathrm{AuV}$ chose their limits to account for driving comfort $[59,60]$. To ensure safe driving in traffic, i.e., avoiding collisions with other vehicles, the minimum gap constraint was imposed as

$$
x_{s-1}(k)-x_{s}(k)-L=g_{s}(k) \geq g_{s, \min }(k)=g_{0}+h_{0, s} v_{s}(k) .
$$

Here, $\Delta g_{s}$ is the gap between the subject vehicle and leading vehicle, $\Delta g_{s, \text { min }}$ is the minimum allowable gap for the subject vehicle, $g_{0}$ is the minimum gap in a vehicle at standstill condition $(2.5 \mathrm{~m})$, and $h_{0, s}$ is the desired headway of the subject vehicle. The following nonlinear constraint was introduced to avoid collision risk during lane changing:

$$
\begin{aligned}
g_{t, \text { lead }} & \geq \theta_{t}(k) \times \Delta g_{s, \text { min }}(k), \\
g_{t, \text { lag }} & \geq \theta_{t}(k) \times \Delta g_{s, \text { min }}(k), \\
g_{t, \text { lead }}(k) & =x_{t-1}(k)-x_{s}(k)-L, \\
g_{t, \text { lag }}(k) & =x_{s}(k)-x_{t}(k)-L .
\end{aligned}
$$

Here, $x_{t-1}$ and $x_{t}$ are the position of lead and lag vehicles, respectively, in the target lane, $L$ is the average length of the vehicle $(5 \mathrm{~m})$, and $\theta_{t}$ denotes current lanes of target lane vehicle and subject vehicle. If the target lane vehicle and the subject vehicle are in the same lane, then $\theta_{t}=1$; otherwise, $\theta_{t}=0$. This time-varying gap constraint defines the permissible gap for lane changing. $\Delta g_{s, \min }$ for $\mathrm{HuVs}$ were measured by taking safety headway value same as desired headway of the particular vehicle. For the cases of lane changing by any $\mathrm{AuV}, \Delta g_{s, \text { min }}$ is measured with $1.25 \mathrm{sec}$ as safety headway. Finally, the state of all vehicles in the simulation was updated by the following equations:

$$
\begin{aligned}
& a_{(s-1) / t /(t-1)}(k)=f\left\{\Delta g_{(s-1) / t /(t-1)}(k), v_{(s-1) / t /(t-1)}(k), \Delta v_{(s-1) / t /(t-1)}(k)\right\}, \\
& \delta_{(r / l), s-1}(k) \\
& \operatorname{or} \delta_{(r / l), t-1}(k), \\
& \operatorname{or} \delta_{(r / l), t}(k)=0 .
\end{aligned}
$$

Here, the first equation was used to estimate the acceleration of surrounding vehicles. The second equation implies that the subject vehicle assumes no other surrounding vehicle is changing lane at any timestep $k$. The acceleration of $\mathrm{HuVs}$ was updated by an enhanced Intelligent Driver Model (IDM) [61], and the model proposed by $\mathrm{Hu}$ et al. [62] was employed to determine the acceleration of AuVs: 


$$
a_{s}(k)=\left\{\begin{array}{l}
a_{\max }\left[1-\left(\frac{v_{s}(k)}{v_{\mathrm{SL}}}\right)^{4}-\left(\frac{g_{0}+\max \left[0, v_{s}(k) \times h_{0}+((v(k) \times \Delta v(k)) / 2 \sqrt{a b})\right]}{s}\right)^{2}\right], \\
k_{1}\left(g_{s}(k)-v_{s}(k) \times h_{0}-g_{0}\right)+k_{2} \Delta v_{s}(k) .
\end{array}\right.
$$

Through equation (7), it was assumed that the vehicles surrounding the subject vehicle (i.e., leading vehicle in the current lane $x_{s-1}$, leading vehicle in the target lane $x_{t-1}$, and the following vehicle in the target lane $x_{t}$ ) are not changing lanes at any timestep $k$. The predicted states of all vehicles for ensuring the safety in the prediction horizon needed to be estimated using

$$
S(k)=\left\{S_{N}(k)\right\}_{\forall N \neq 0},
$$

which denotes $S$ as the state of traffic considering $S_{N}$ as the estimated state of the individual vehicle where
$S_{N}(k)=\left\{\widehat{s}_{N}(k), \ldots, \widehat{s}_{N}(k+P)\right\}$. Since the state vector of the individual vehicle included time-varying continuous variables (e.g., position, velocity, current, and destination lane of the vehicle) and an integer discrete variable, the optimization of both longitudinal and lateral control decisions became computationally demanding and uncertain in obtaining optimal solution within a finite horizon. Therefore, based on the control requirement (i.e., longitudinal or lateral) at a timestep, respective control optimization was considered to estimate the state of the vehicles:

$$
\begin{aligned}
\text { longitudinal control: } J_{\text {long }} & =\sum_{i=k}^{k+P}\left[v_{s}(i)-v_{\text {des }}\right]^{2}+\sum_{i=k}^{k+P} a_{s}^{2}(i), \\
\text { lateral control: } J_{\text {lat }} & =\sum_{i=k}^{k+P}\left[v_{s}(i)-v_{\text {des }}\right]^{2}+\sum_{i=k}^{k+P} a_{s}^{2}(i)+\sum_{i=k}^{k+P} \theta_{t}(i) e^{-\alpha_{t}(i)\left(\left(g_{t, \text { lead }}^{2}(i)+g_{t, \text { lag }}^{2}(i)\right) / 2\right) .}
\end{aligned}
$$

Here, $v_{\text {des }}$ is the desired velocity equal to the speed limit (i.e., $25 \mathrm{~m} / \mathrm{s}$ ). The two terms in longitudinal control ensure the vehicles drive close to the desired velocity with little or no acceleration. 1st term in equation (9) compelled the vehicles to attain the desired velocity to minimize the objective function. 2nd term of longitudinal control restricts blunt fluctuation of acceleration while in motion. Both these aspects are important during the car-following state. Equation (10) considered the additional condition of an acceptable gap in the target lane while changing lanes. While the 1 st and 2 nd terms in equation (10) were the same as equation (9), the third term in lateral control cost function (equation (10)) penalizes for unsafe lane changes in the form of Gaussian function. The value of coefficient $\alpha_{t}$ was measured according to Kamal et al. [17], which defines the shape of the Gaussian function. Furthermore, planning for a lane change near the end of a horizon limit is not preferred. Since it is more likely that the predicted states of the other vehicles vary in the course, this may force the vehicle to give up on a lane change process before completion. Specifically, minimum steps to initiate a lane change can be imposed by suitably choosing $\delta$. Once the vehicle is on course to lane change, the receding horizon approach is applied for successively relaxing $\delta$. Finally, a lane change is executed in a predefined reference trajectory.

The subject vehicle was provided with two sets of data at each timestep: (i) vehicle's status memory and (ii) environment inputs. The vehicle's status memory included acceleration, velocity, position, yaw rate, lane position, and destination lane information of the subject vehicle for the final 50 timesteps. Environment inputs included velocity, position, and lane position vehicles surrounding the subject vehicle in the weaving section. With those inputs, the subject vehicle decided between longitudinal and lateral control to update the vehicle status. Regarding the driver's control decisions, both longitudinal and lateral control decisions contained two states. When only the longitudinal control decision was active, the subject vehicles could be either in the free-flow or car-following state. The prerequisites of these states are listed as follows:

(i) Free-flow state was triggered when the upstream area of the vehicle is empty, or more than enough safety gap was available for the vehicle to drive at $10 \%$ higher than the speed limit.

(ii) Car-following state was activated when there was spatial constraint due to the presence of a leading vehicle that restricted the vehicle to drive under the speed limit.

For $\mathrm{HuVs}$, a discretionary lane change was initiated when the vehicle drove at $10 \%$ lower than the speed limit for $5 \mathrm{sec}$. The discretionary lane-changing actions by $\mathrm{HuVs}$ were motivated by driving at the speed limit. AuVs sought lanechanging opportunities due to the possibility of forming platoons with the leading vehicle in the target lane. The lateral control state was active when the subject vehicle attempts to change lanes. Discretionary lane changing was omitted for the weaving section in this part of the research. 
Due to the inherent characteristics of weaving section traffic flow patterns, it was assumed that the likelihood of vehicles performing discretionary lane changing in this roadway section would be insignificant. Therefore, only mandatory lane-changing occurred based on the destination lane assigned for the vehicle and the vehicle looking for lanechanging opportunities as soon as it enters the weaving section. In the lateral control state, there were two possible scenarios:

(i) Initiate was active when enough gap was available in the target lane to change lane safely.

(ii) Terminate became active when the available gap in the target lane was not enough to execute lane changing safely. In this case, the vehicle was forced to remain in its lane.

Figure 1 illustrates the individual vehicle control process at every timestep. The subject vehicle was programmed to search for a lane-changing opportunity to initiate mandatory lane-changing before it terminates the search due to lack of available lead and lag gap in the target lane. The subject vehicle could accept up to the minimum gap between vehicles in standstill conditions $\left(\mathbf{g}_{0}\right)$ to execute mandatory lane changing as it approached the end of the weaving section. Lane-changing duration and reference trajectory for modeling was calibrated by analyzing 631 events of successful lane-changing in freeway from naturalistic driving data obtained from the Safety Pilot Model Deployment (SPMD) project [63] database. The identified mean lane-changing duration, irrespective of lane-changing type, was $2.3 \mathrm{sec}$. The vehicle's center of gravity moving from the middle of the current lane to the middle of the target lane constituted the duration measurement. A reference trajectory was developed for all vehicles to follow during lane-changing maneuvers. When the vehicle decided to initiate lane changing through MPC-based lateral control considering the relative gap in the target lane, it followed the reference trajectory over the lane-changing duration $(2.3 \mathrm{sec})$. Both $\mathrm{HuVs}$ and $\mathrm{AuVs}$ would follow this trajectory for lane-changing purposes. Behavioral implications of $\mathrm{HuVs}$ on lane-changing maneuver had not taken into account in this research. Although the literature has suggested a higher lane-changing duration for freeways [64-67], most of these measurements include waiting time before lane changes to find suitable gaps. Since this model considered the 5-sec time window for evaluating lane-changing warrants, the smaller active lanechanging duration is justified.

\section{Model Validation}

According to the Highway Capacity Manual (HCM), the weaving section length varies between $150 \mathrm{~m}$ and $750 \mathrm{~m}$. The studied roadway segment was designed as a 600-m long twolane Type-A weaving section of a freeway. The speed limit of the roadway was taken to be $25 \mathrm{~m} / \mathrm{s}$ ( $90 \mathrm{kph}$ ). The lanechanging vehicle ratio (VR) was assigned to be $10 \%$ of the flow rate. The destination lane of individual vehicles was assigned randomly during the entry of the simulated roadway segment. The agent-based modeling approach was taken for simulating different inflow rates and $\mathrm{AuV}$ shares, which provides the opportunity to investigate complex interactions between two distinct groups of vehicles. HCM suggests that the weaving flow rate should not exceed 2800 vehicle per hour per lane (vphpl) for a Type-A weaving section as a higher inflow rate would be prone to more frequent operational failure. Although this restriction on inflow rate was imposed specifically for roadways containing only $\mathrm{HuVs}$, the maximum inflow rate of the simulation model was kept at $2800 \mathrm{vphpl}$ since no specific instruction was provided for mixed traffic inflow rates in HCM. Since it was not established before the analysis that AuVs could effectively increase the vehicle movement capability of the roadway segment, increasing inflow rates over the suggested level could induce operational failure due to high inflow rates. Hence, the simulated flow rates were restricted from $1200 \mathrm{vphpl}$ to $2800 \mathrm{vphpl}$ with $100 \mathrm{vphpl}$ increment. The varying inflow rate was simulated by adjusting the average headways of vehicle entrance for both types of vehicles. AuV market share was maintained by regulating the number of $\mathrm{AuVs}$ present at a time during the simulation. However, each inflow rate and $\mathrm{AuV}$ share scenario was simulated 20 times to offset the randomness of vehicle entrance pattern and human driving behavior in the simulation.

To validate the proposed traffic model, the traffic data generated by the model were compared to the standard used in HCM. Numerous simulations were run, having only $\mathrm{HuV}$ scenarios for varying inflow rates; the macroscopic parameters (i.e., flow, density, and space mean speed) were recorded. All these recorded data points were then plotted to develop the fundamental diagram (i.e., flow-density diagram) and determine the segment capacity of the simulated weaving section. As illustrated in Figure 2(a), the fundamental diagram of the model weaving section was generated from numerous passes of the developed model. Figure 2(b) was generated from HCM that illustrated the three-lane Type-A weaving section's confirmed capacity with a $100 \mathrm{kph}$ free-flow speed. The capacity term was defined in HCM as the maximum number of vehicles that can pass a given point under prevailing roadway, traffic, and control conditions. Following the definition, the capacity of the simulated section was identified as $2088 \mathrm{vph}$ from the flow-density diagram. Comparing the simulated weaving section's obtained capacity with Figure 2(b) demonstrated that the capacity value falls near the capacity curve for $\mathrm{VR}=0.10$ of the three-lane weaving section with $600 \mathrm{~m}$ length. Hence, the developed model was deemed to be consistent with realworld roadway and traffic scenarios.

To further consolidate the model validity, the Level of Service (LOS) criteria established for the freeway weaving segment in HCM was matched with obtained model output. As stated in HCM, the capacity of a weaving segment is the result of flows that causes the density to reach Level of Service (LOS) E/F for freeways. Table 2 outlines the different lane density ranges reported in HCM for the distinct LOS of weaving sections in freeways. The model-generated fundamental diagram exhibited the capacity at $23.81 \mathrm{vpkpl}$ density level. According to Table 2, this density value falls under LOS 


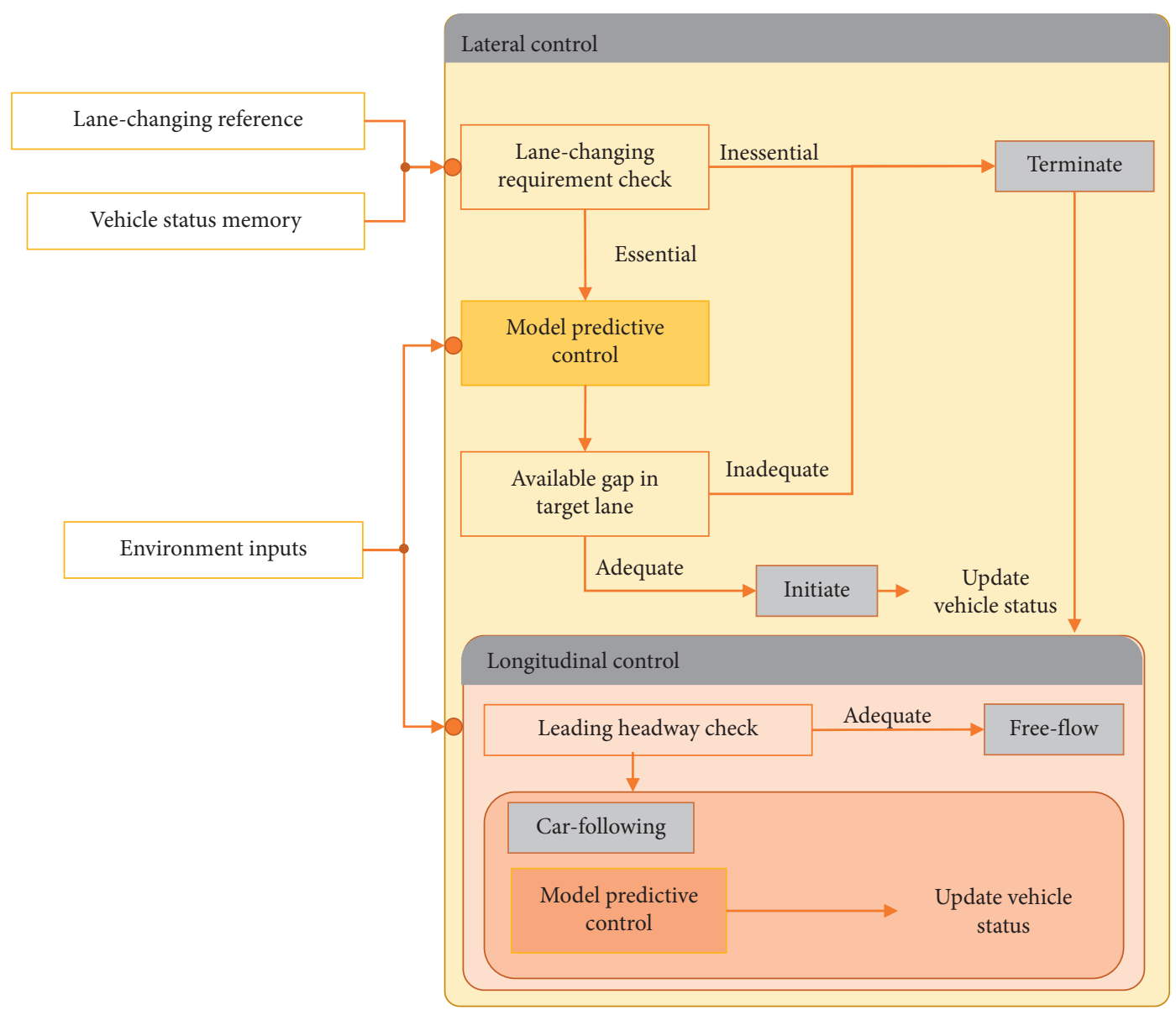

FIgURE 1: Control decision process of an individual vehicle at each timestep.

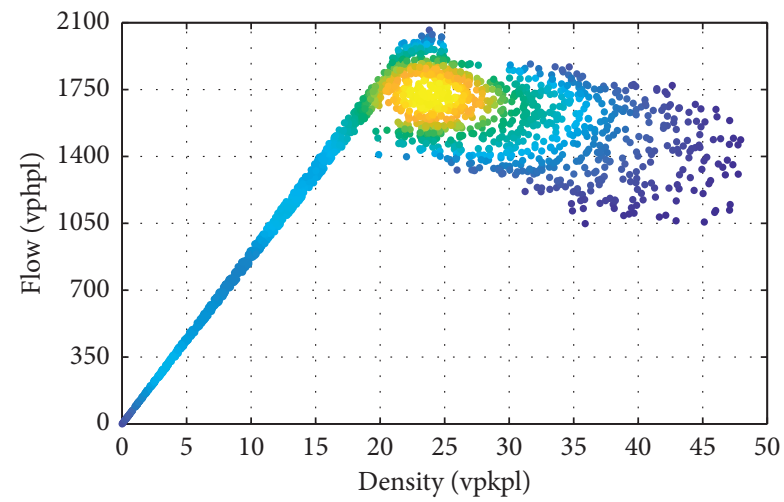

(a)

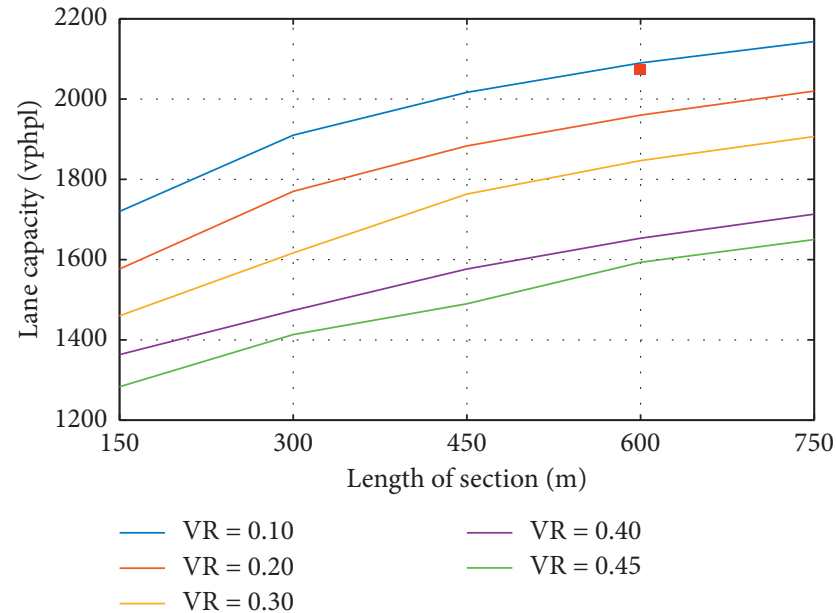

(b)

FIgURE 2: (a) Flow-density diagram from simulation runs with HuVs only; (b) lane capacity at varying length and VR of weaving section.

E for freeway weaving sections and therefore complies with the requirement stated in HCM. Since the results from both analyses were consistent with the expected outcome, the developed microscopic model was regarded as an effective representation of real-world traffic and could therefore be applied for future analysis.

\section{Mobility Implications}

The mobility consequences resulting from mixed traffic movement were measured considering two key parameters: (i) maximum throughput; (ii) average speed of traffic. The inflow rate varied from 1200 to $2800 \mathrm{vphpl}$ with $100 \mathrm{vphpl}$ 
TABLE 2: Level of Service (LOS) criteria for weaving segments.

\begin{tabular}{lcc}
\hline LOS & Lane density (vpk) \\
& Multilane and collector-distributor weaving section \\
\hline A & $\leq 6.0$ & $\leq 8.0$ \\
B & $>6.0-12.0$ & $>8.0-15.0$ \\
C & $>12.0-17.0$ & $>15.0-20.0$ \\
D & $>17.0-22.0$ & $>20.0-23.0$ \\
E & $>22.0-27.0$ & $>23.0-25.0$ \\
F & $>27.0$ & $>25.0$ \\
\hline
\end{tabular}

increments in each step. Similarly, the AuV share was increased from $5 \%$ to $95 \%$, with a $5 \%$ increment in each step. As a result, 323 unique scenarios of mixed traffic movement were generated. To account for the fluctuations resulting from driving behavioral variations of human drivers and AuVs' location and distribution on measured parameters, each simulation scenario (i.e., inflow rate and $\mathrm{AuV}$ share) was repeated 20 times. The analyzed outcomes of these scenarios were compared with the base case ( $0 \% \mathrm{AuV}$ share) to compute the consequences of mixed traffic movements. To calculate the chosen mobility parameters of each scenario, the simulation period (18000 timesteps $=30$ minutes) was divided into six 5-min intervals, and the maximum/ average parameter value was measured for each interval. Finally, the maximum parameter value was chosen by taking the maximum interval values for maximum throughput and average interval values for the average traffic speed.

5.1. Maximum Throughput. While much effort has been made to specify the impact of AuVs on the maximum throughput of freeways [9, 20, 24-26, 34, 68-72], the variability in results kept it as an open and debated question. Hence, this study attempts to confront the issue with a precise scope of estimation for Type-A weaving sections with a $90 \mathrm{~km} / \mathrm{h}$ speed limit and restricted inflow rates. The simulated scenarios varied the AuV shares from 5\% to $95 \%$ to maintain a mixed traffic environment and compared the performance with a base case to appraise potential changes. As illustrated in Figure 3, the variation in maximum throughput is nonlinear and much greater due to variations in $\mathrm{AuV}$ share than inflow rate changes. Maximum detriment in throughput was experienced at $10 \% \mathrm{AuV}$ share $(-3.88 \%$, 1981 vphpl@1400vphpl inflow rate) and maximum gain was attained at 65\% AuV share (80.49\%,3720vphpl@2200vphpl inflow rate) in comparison to base case (2061 vphpl). We compared our attained results with Tilg et al. [34], where they evaluated the effects of automated vehicles in freeway weaving sections. Their study found that the maximum throughput of the simulated weaving section increased from 1700vphpl@0\% AuV to 3300vphpl@100\% AuV (94.12\%). Although a comparable level of increase was observed in this study, the maximum increase in throughput was observed at a lower AuV share (65\%). Recently, Rezaei and Caulfield [73] drew similar conclusions from their study where optimal mobility was achieved for both off-peak and peak traffic scenarios at $60 \% \mathrm{AuV}$ shares. Attained maximum throughput for increasing $\mathrm{AuV}$ shares was further compared with the analysis of Liu et al. [68], which estimated the theoretical capacity of homogenous freeway sections resulting from mixed traffic. The following equation was applied to calculate the theoretical capacity at varying $\mathrm{AuV}$ shares:

$$
\begin{aligned}
Q_{\mathrm{max}} & =\frac{3600}{P_{\mathrm{AuV}(l)} \times \mathrm{HW}_{\mathrm{AuV}(l)}+P_{\mathrm{AuV}(f)} \times \mathrm{HW}_{\mathrm{AuV}(f)}+P_{\mathrm{HuV}} \times \mathrm{HW}_{\mathrm{HuV}}}, \\
P_{\mathrm{AuV}(f)} & =\delta \times \delta \\
P_{\mathrm{AuV}(l)} & =\delta(1-\delta), \\
P_{\mathrm{HuV}} & =1-P_{\mathrm{AuV}(f)}-P_{\mathrm{AuV}(l)} .
\end{aligned}
$$

Here, $P_{\mathrm{AuV}(l)}, P_{\mathrm{AuV}(f)}$, and $P_{\mathrm{HuV}}$ are the probability of any vehicle being $\mathrm{AuV}$ platoon leader, follower, and $\mathrm{HuV}$, respectively; $\mathrm{HW}_{\mathrm{AuV}(f)}, \mathrm{HW}_{\mathrm{AuV}(l)}$, and $\mathrm{HW}_{\mathrm{HuV}}$ are average safety headways of AuV platoon leader (i.e., $1.25 \mathrm{sec}$ ), follower (i.e., $1.0 \mathrm{sec}$ ), and $\mathrm{HuV}$ (i.e., $1.5 \mathrm{sec}$ ), respectively; $\delta$ is $\mathrm{AuV}$ share. Comparison between the theoretical capacity and the highest maximum throughput value for specific $\mathrm{AuV}$ share displayed a similar pattern up to $65 \% \mathrm{AuV}$ share (Figure 3(b)). While theoretical capacity showed an increase, the simulation results demonstrated a steady decline. This phenomenon can be explained by the fact that our inflow rate was bounded at $2800 \mathrm{vphpl}$ based on the HCM guideline restricting potential gains. Additionally, in the simulation, both vehicle types were designed to lane change with the same trajectory with a similar acceptable gap and safety headway conditions that could play a critical role in restricting maximum throughput after a threshold $\mathrm{AuV}$ share. At higher $\mathrm{AuV}$ shares, platoons formed by $\mathrm{AuVs}$ 


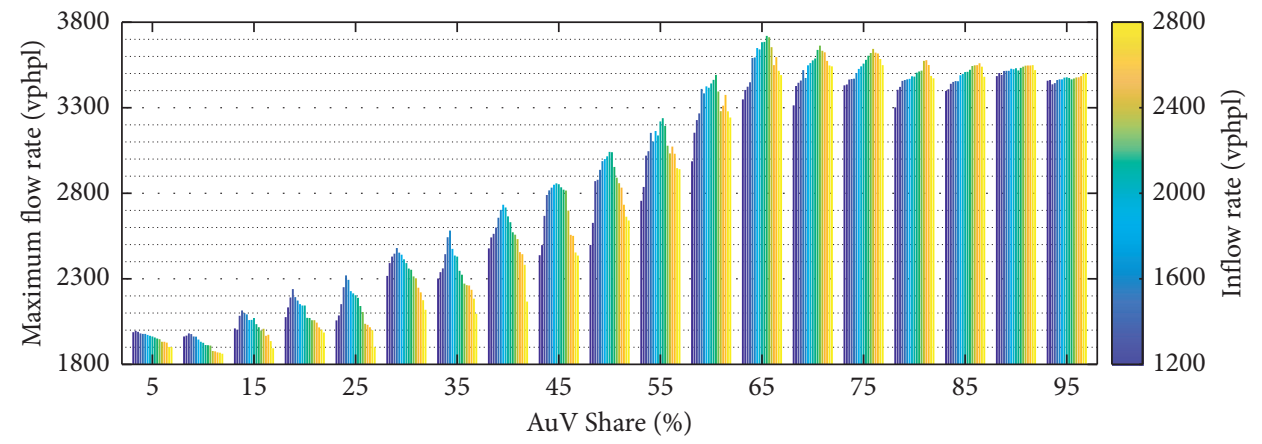

(a)

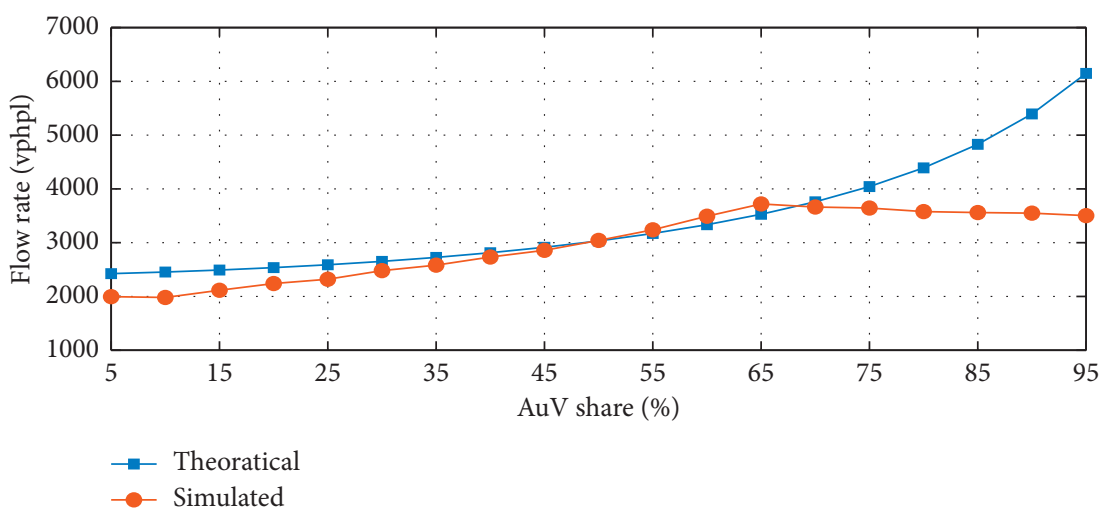

(b)

FiguRE 3: (a) Maximum flow rate through weaving section at various traffic state; (b) comparison between theoretical and simulated maximum flow rate for mixed traffic.

reduced lane-changing scopes for the remaining $\mathrm{HuVs}$ and forced them to create larger gaps between platoons to execute mandatory lane changes in weaving sections.

Analysis of maximum throughput fluctuations due to varying inflow rates at a specific $\mathrm{AuV}$ share identified the highest variations at $45 \% \mathrm{AuV}$ share, which gradually receded with increased $\mathrm{AuV}$ shares. In addition to behavioral variations of human drivers, represented by the desired headway parameter of $\mathrm{HuVs}$, the competing lane-changing opportunity searches by both vehicle types reached the maximum level once the traffic achieved equitable shares of $\mathrm{AuVs}$ and $\mathrm{HuVs}$, which explains the highest variation at $45 \%$ $\mathrm{AuV}$ share and lower variations at higher as well as lower $\mathrm{AuV}$ shares.

5.2. Average Speed of Traffic. One of the key indicators of mobility is the average speed of traffic. An increase in the average traffic speed can be interpreted as a reduction in travel time and vice versa. Furthermore, an experimental study conducted by Jiang et al. [74], specifically with AuVs forming a 51-vehicle platoon, indicated that traffic instability could be better reported through average traffic speed than traffic density or spacing. In this regard, the average space mean speed of the weaving section was measured for each simulated scenario. Figure 4 illustrates the obtained average speed outcomes for different simulated scenarios. As observed in Figure 4(a), the average traffic speed resulting from various inflow rates was highly dispersed with lower $\mathrm{AuV}$ shares (5-20\%). Each black dot for a specific AuV share represents the average speed at a particular inflow rate. The average speed value of a specific scenario (i.e., inflow rate and $\mathrm{AuV}$ share) was obtained by taking the average speed of all the repeated simulation runs for that scenario.

On the other hand, with increasing $\mathrm{AuV}$ shares, the resultant average speed became increasingly concentrated, shifting towards the speed limit. The dispersion of average speed at lower AuV share indicated instability in traffic flow resulting from sparsely spaced AuVs in traffic. The instability of traffic at lower $\mathrm{AuV}$ shared was also translated into lower maximum throughput at similar $\mathrm{AuV}$ share, as presented in the final section. However, with gradual increase of AuVs brought more stability, which was perceivable from a more compact average speed distribution of traffic. Figure 4(b) provides a more explicit demonstration of the interaction between inflow rate and $\mathrm{AuV}$ shares with the average traffic speed. Although the average traffic speed changes at the lower inflow rate were negligible, reductions in the average speed at lower $\mathrm{AuV}$ shares were prominent at high inflow rates. Comparing mixed traffic scenarios with base case average speed (82.75 kph) obtained a maximum reduction of $11.53 \mathrm{kph}$ at $20 \% \mathrm{AuV}$ share and $2700 \mathrm{vphpl}$ inflow rate and a maximum increase of $7.22 \mathrm{kph}$ at $95 \% \mathrm{AuV}$ share and $2400 \mathrm{vphpl}$ inflow rate. It should be noted that both types of vehicles were simulated to attain the desired speed, which was the speed limit $(90 \mathrm{kph})$. Therefore, the maximum possible increase in average speed was $7.25 \mathrm{kph}$ within the design framework. 


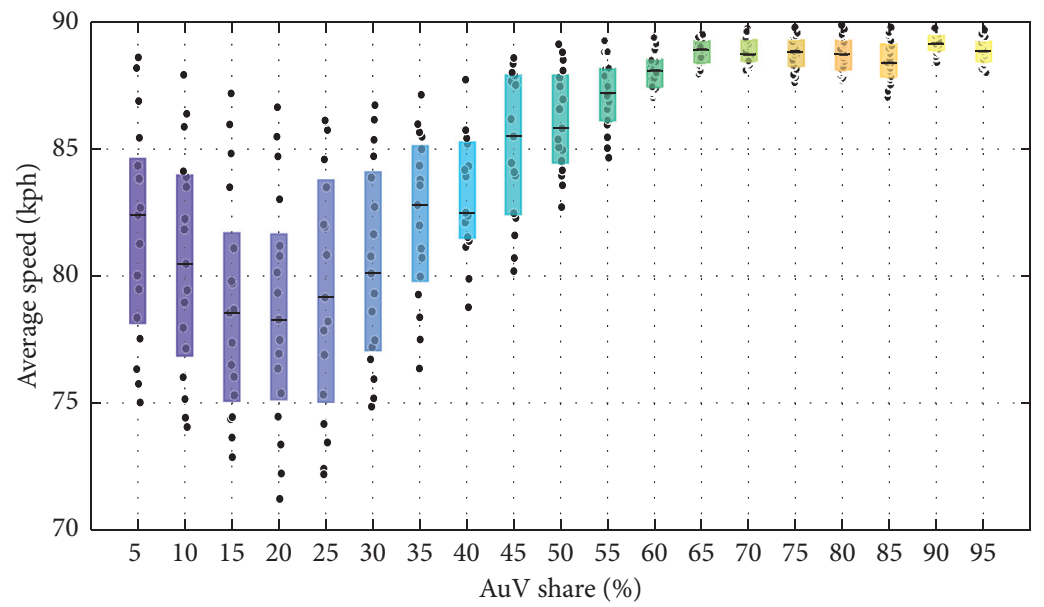

(a)

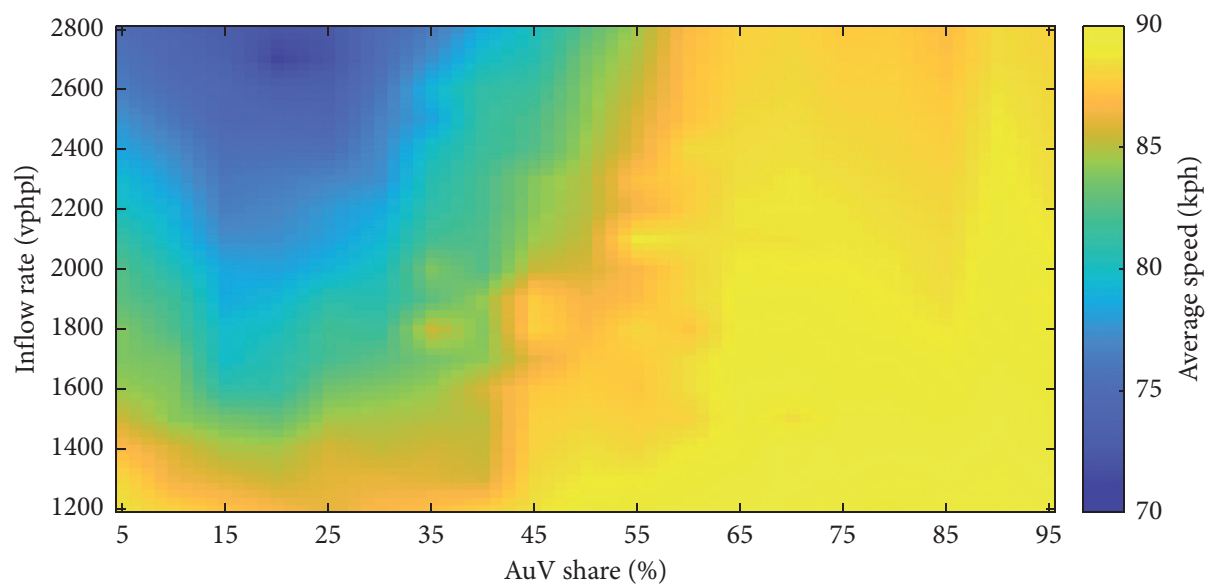

(b)

FIGURE 4: (a) Average speed of weaving section for simulated mixed traffic scenarios; (b) average speed heatmap for varying inflow rates and $\mathrm{AuV}$ shares.

Liu et al. [68] noted a minor improvement at a lower market share as compared to a drastic improvement at a higher market share. However, the study considered market share at $20 \%$ increments, limiting the understanding for $\mathrm{AuV}$ shares with more nuanced increments. In contrast to Liu et al., the findings from this study showed slower traffic movements at lower $\mathrm{AuV}$ shares in high traffic demand. These events could have been triggered by the dispersed positioning of AuVs in traffic that could not form closely spaced CACC platoons with other AuVs. As a result, these isolated $\mathrm{AuVs}$ had to maintain ACC driving principles while accommodating lane-changing vehicles at high inflow rates. Tilg et al. [34] mentioned that these gap-searching vehicles, specifically $\mathrm{HuVs}$, related to speed attenuation until they engaged in successful lane-changing maneuvers. The gradual increase of the $\mathrm{AuV}$ share improved platoon forming probability that led to more stable traffic movement and is analogous to the findings by Talebpour and Mahmassani [75]. Those authors demonstrated the instability in traffic flow resulting from lower connected and automated vehicle shares. On the other hand, Spiliopoulou et al. [76] concluded that almost all congestion of the studied corridor could be eliminated at $60 \%$ market penetration. Our study also demonstrated a similar pattern since the average speed over the simulation duration of most scenarios beyond $60 \%$ market share was close to the speed limit.

\section{Safety Implications}

Traffic safety assessment is vital when analyzing unfamiliar transportation system performance. The developed microscopic simulation framework provides the opportunity to perform a detailed safety implication analysis on simulated mixed traffic scenarios without physically implementing the $\mathrm{AuVs}$ in a transportation system. However, two major limitations of the simulation models are (1) the discounting of collision events among simulated vehicles and (2) the inability to simulate human drivers' distraction and misjudgement errors. Hence, the safety analysis of simulated models is dependent on interpreting and comparing surrogate measures of safety. In this research, we have used time-to-collision (TTC) as the primary measure of effectiveness for safety as its use in this capacity has significant precedence in the literature. Safety-critical TTC events were detected from simulated 
scenarios with maximum throughput and then translated into relatable safety parameters such as potential conflict events and rear-end crash potential.

There are four types of vehicle interaction scenarios in a mixed traffic environment: $\mathrm{HuV}$ with an $\mathrm{HuV}$ (potential) leader $(\mathrm{HuV}-\mathrm{HuV}), \mathrm{HuV}$ with an $\mathrm{AuV}$ (potential) leader $(\mathrm{HuV}-\mathrm{AuV}), \mathrm{AuV}$ with an $\mathrm{HuV}$ (potential) leader (AuV$\mathrm{HuV}$ ), and $\mathrm{AuV}$ with an $\mathrm{HuV}$ (potential) leader ( $\mathrm{AuV}-\mathrm{AuV}$ ). However, any commonly accepted threshold of surrogate safety measures has yet to be established for $\mathrm{AuV}$ in the literature. Hence, in this study, we only examined the interactions of $\mathrm{HuV}$ with other $\mathrm{HuV}$ and $\mathrm{AuV}$ leaders for potential conflict identification. Since the market share of $\mathrm{AuVs}$ changes with different scenarios, the crash risk rate for $\mathrm{HuVs}$ and conflict frequencies are used to identify the safety implications of AuVs in the mixed traffic environment.

6.1. Potential Conflict Events. TTC, defined as the expected time before two vehicles collide if they remain on the same path at the same speed, is a widely used surrogate safety measure. The Surrogate Safety Assessment Model [77] suggests $1.5 \mathrm{sec}$ TTC as the threshold value for identifying potential safety concerns within a simulation environment, adopted in our research to extract the number of possible conflict events from simulated scenarios. The identified possible conflict events were classified into two collision groups: (i) rear-end and (ii) lane changing. The driving state of the subject vehicle was checked to classify the potential conflict type. If the TTC value fell below the threshold during the free-flow or car-following longitudinal control state, it was classified as a rear-end conflict event. On the other hand, if the TTC value fell below the threshold during the initial lateral control state, it was classified as a lanechanging conflict event. The following equations were used to measure and identify a potential conflict event:

$$
\begin{aligned}
\mathrm{CE}_{s}(k) & = \begin{cases}1, & \text { if } \mathrm{TTC}_{s}(k) \leq 1.5 \\
0, & \text { otherwise, }\end{cases} \\
\operatorname{TTC}_{s}(k) & =\frac{x_{s-1}(k)-x_{s}(k)-L}{v_{s}(k)-v_{s-1}(k)}=\frac{g_{s}(k)}{v_{s}(k)-v_{s-1}(k)} .
\end{aligned}
$$

Here, $\mathrm{CE}_{s}(k)$ is the conflict event count of the subject vehicle, $s$ at timestep $k . x_{i}, v_{i}$, and $g_{i}$ represent $i$ vehicle's position, velocity, and lead gap (current lane), respectively. Since the simulation was repeated several times for specific traffic flow scenarios (i.e., inflow rate and $\mathrm{AuV}$ share), the average number of conflict events was reported for each particular scenario. The aim was to reduce the stochastic effects resulting from the behavioral variation of $\mathrm{HuVs}$ and placement changes of AuVs. Since the threshold TTC value for identifying the conflict events was lower than the desired headway of AuVs (i.e., ACC $=1.25 \mathrm{sec}$ and CACC platoon $=1.0 \mathrm{sec}$ ), the conflict events that emerged from AuVs were disregarded from the total conflict event computation. Hence, the identified conflicts were generated exclusively from $\mathrm{HuVs}$ interacting with other $\mathrm{HuVs}$ and $\mathrm{AuVs}$ in the vicinity.

Figures 5(a)-5(f) show the pattern of traced potential conflict events at varying traffic states. It is evident that as the $\mathrm{AuV}$ share increased, there was a clear and significant decrease in conflict event counts. The summation of potential conflict events, irrespective of inflow rates, was $94.28 \%$ lower at $95 \% \mathrm{AuV}$ share (186 conflict events; 42 rear-end, 144 lanechanging) in comparison to the base case (3249 conflict events; 1238 rear-end, 2011 lane-changing). The gradual decline of conflict events experienced an exponential pattern with $\mathrm{AuV}$ share expansion (Figure 5(g)). While larger shares of conflicts were found to be lane-changing (base case: $38.1 \%$ rear-end, $61.9 \%$ lane-changing), the presence of AuVs was relatively more proficient in mitigating rear-end type conflicts (95\% AuV share: $22.6 \%$ rear-end, $77.4 \%$ lane-changing) by $\mathrm{HuVs}$.

The potential conflict events were further explored by the proposed methods of $\mathrm{Oh}$ and Kim [78] to estimate the crash risk index (CRI) from the TTC values in the analyzed roadway segment. While it was possible to obtain the deterministic control state of each vehicle under the current modeling framework, the probabilistic estimation of lanechanging decisions was retained for the novelty of the proposed method. The following equations were used to determine the crash risk index of a specific traffic scenario:

$$
\begin{aligned}
P\left(\mathrm{Cr}_{s}\right)(k) & =P\left(\mathrm{NLC}_{s} \mid X_{s}\right)(k) \times P\left(\mathrm{NLC}_{s-1} \mid X_{s-1}\right)(k) \times P\left(C_{s} \mid \mathrm{TTC}_{s}\right)(k), \\
P\left(\mathrm{NLC}_{s}=1 \mid X_{s}\right)(k) & =\frac{\exp \left[f\left(X_{s}, \beta\right)\right]}{1+\exp \left[f\left(X_{s}, \beta\right)\right]}=\frac{1}{1+\exp \left(11.476-0.045 v_{s}(k)-0.083 v_{t}(k)-0.046 g_{s}(k)+0.023 g_{t}(k)\right)}, \\
P\left(C_{s} \mid \mathrm{TTC}_{s}\right)(k) & =\exp \left(-\frac{1}{c}\left(\frac{g_{s}(k)}{v_{s}(k)-v_{s-1}(k)}\right)\right) \\
P\left(\mathrm{Cr}_{s}\right)(k) & =\left[\frac{\exp \left[f\left(X_{s}, \beta\right)\right]}{1+\exp \left[f\left(X_{s}, \beta\right)\right]}\right] \times\left[\frac{\exp \left[f\left(X_{s-1}, \beta\right)\right]}{1+\exp \left[f\left(X_{s-1}, \beta\right)\right]}\right] \times \exp \left[-\frac{1}{c}\left(g_{s-1}(k) /\left(v_{s}(k)-v_{s-1}(k)\right)\right)\right] \\
\mathrm{CRI}_{i} & =\frac{\sum_{s=1}^{S} \sum_{t=1}^{T} P(\mathrm{Cr})_{s}(k)}{K \times S} .
\end{aligned}
$$




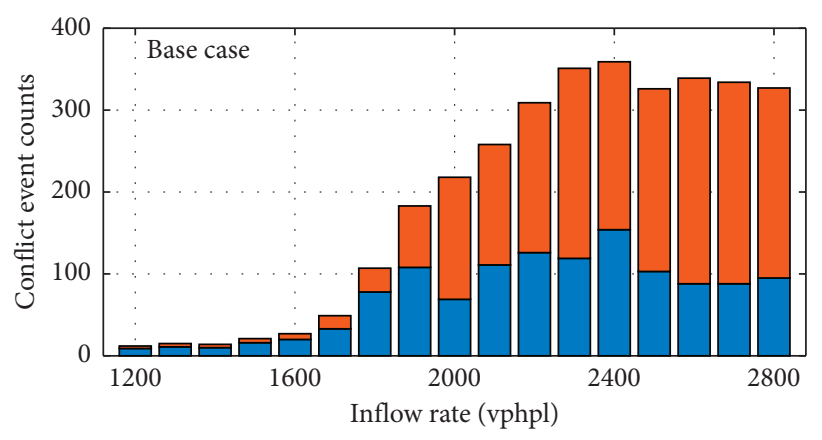

$\square$ Rear-end

$\square$ Lane-changing

(a)

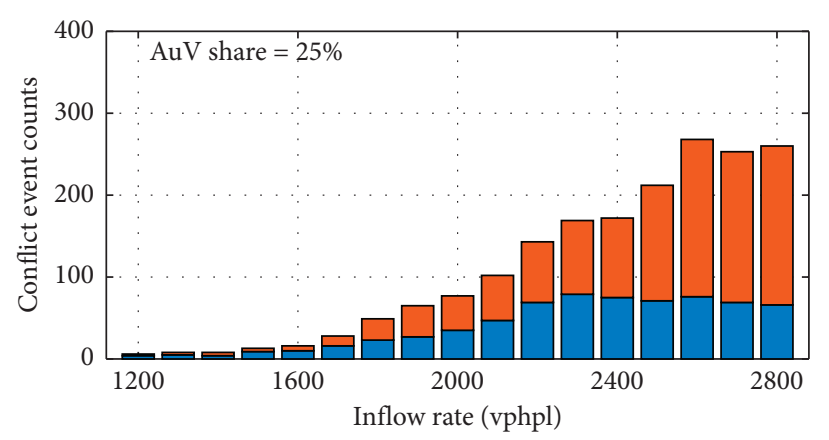

$\square$ Rear-end

$\square$ Lane-changing

(c)

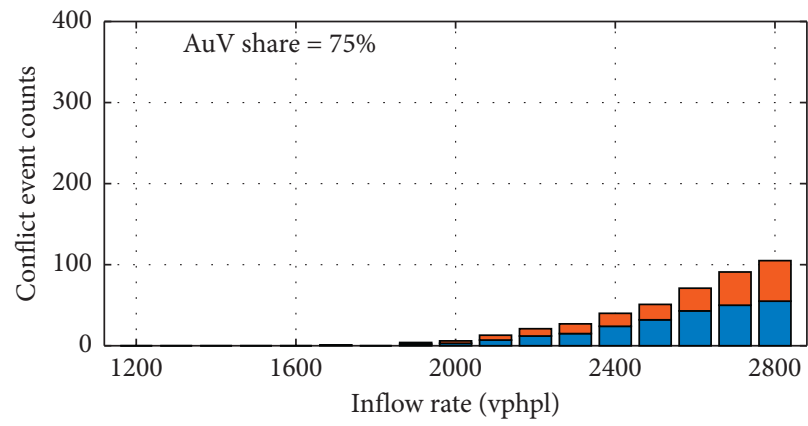

$\square$ Rear-end

$\square$ Lane-changing

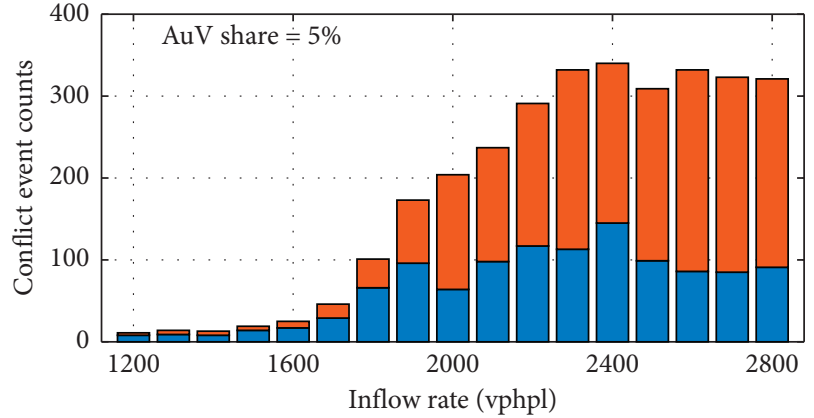

$\square$ Rear-end

$\square$ Lane-changing

(b)

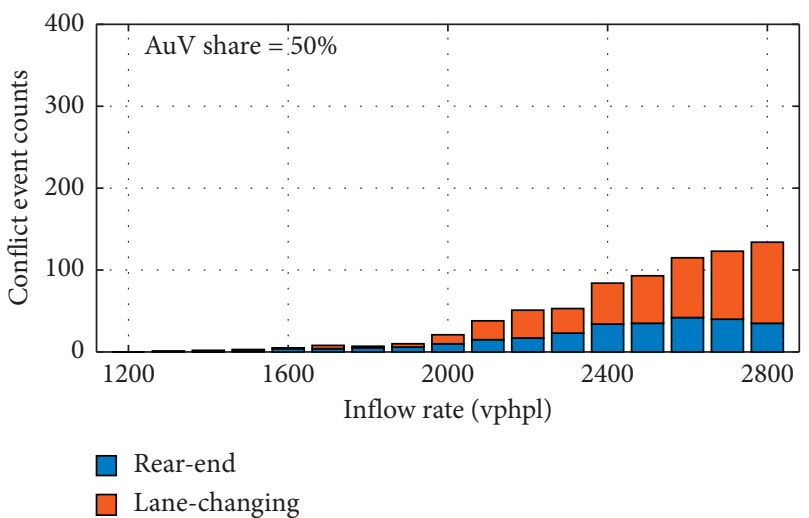

(d)

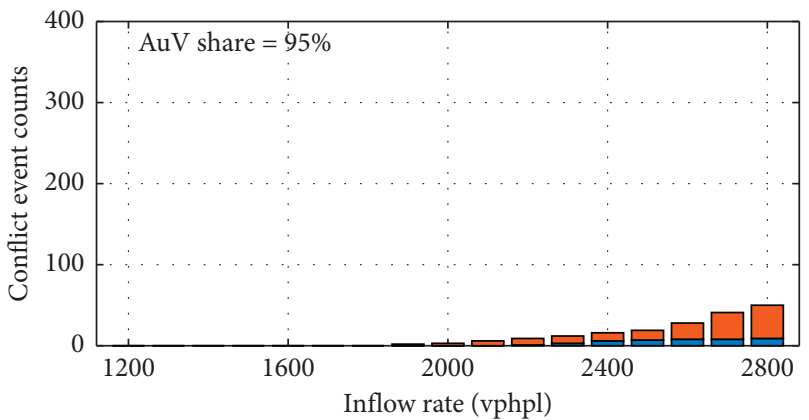

$\square$ Rear-end

$\square$ Lane-changing

(e)

Figure 5: Continued. 


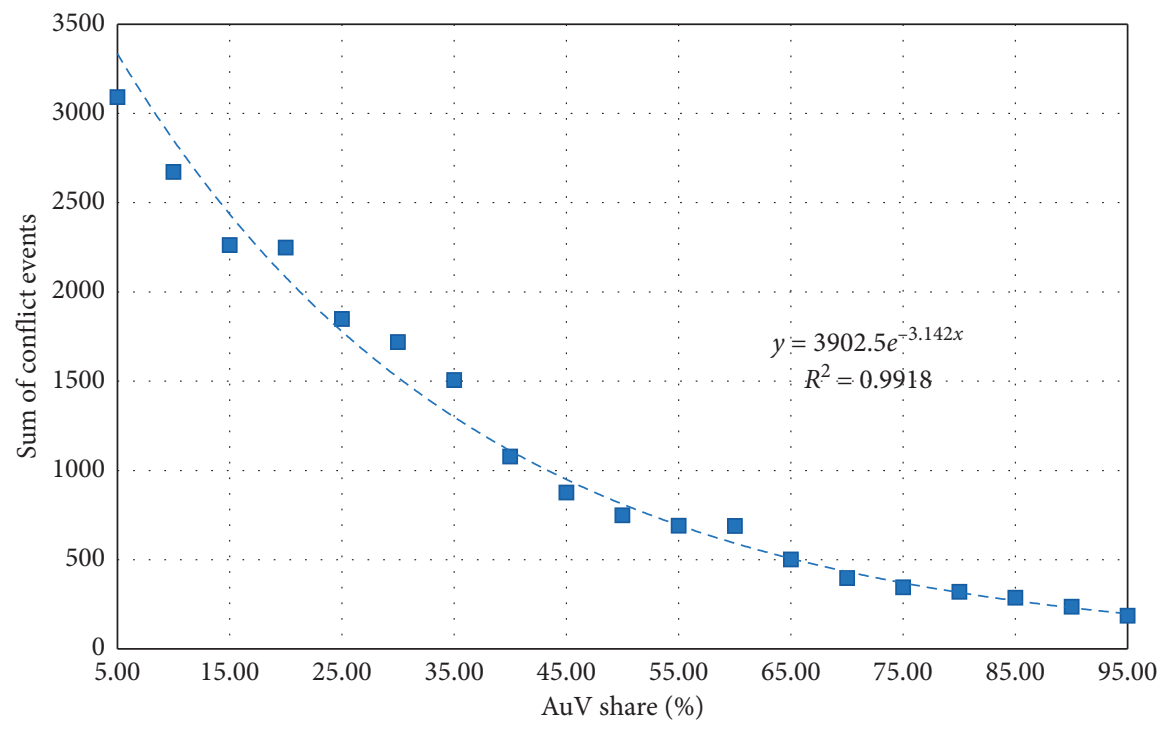

(g)

Figure 5: Counts of detected conflict events at (a) base case (0\%), (b) 5\%, (c) 25\%, (d) 50\%, (e) 75\%, and (f) $95 \%$ AuV share, and (g) pattern of total detected conflict events at increasing AuV share.

Here,

(i) $x_{i}, v_{i}$, and $g_{i}$ represent $i$ vehicle's position, velocity, and lead gap (current lane), respectively

(ii) $P\left(\mathrm{Cr}_{s}\right)$ is the probability that the subject vehicle would be involved in a rear-end crash

(iii) $P\left(\mathrm{NLC}_{s}=1 \mid X_{s}\right)$ is the probability that the subject vehicle will not change lanes (NLC) under adjacent (i.e., $s-1, t, t-1$ vehicles) vehicle conditions (Figure 6(a))

(iv) $P\left(C_{s} \mid \mathrm{TTC}_{s}\right)$ is the probability that the subject vehicle would collide with the front vehicle given the current TTC

(v) $\mathrm{CRI}_{i}$ is the crash risk index of scenario $i$

(vi) $K$ is the total timesteps of analysis

(vii) $S$ is the number of vehicles passing through the weaving section

Further details and parameter values are available in $\mathrm{Oh}$ and Kim [78]. In applying this method, the CRI values for each mixed traffic simulation scenario (i.e., inflow rate and $\mathrm{AuV}$ share) were measured from identified potential conflict events. TTC values more than the threshold TTC (i.e., $1.5 \mathrm{sec}$ ) were discarded from the CRI calculation. For each traffic state (i.e., inflow rate and $\mathrm{AuV}$ share), the simulation instances with maximum numbers of potential conflict events were chosen to measure CRIs by plotting them, as illustrated in Figure 6. As shown in Figure 6(b), the overall CRI showed a nonlinear downward trend with increasing $\mathrm{AuV}$ shares. The maximum CRI was experienced at a $5 \% \mathrm{AuV}$ share (average $=0.0207$, standard deviation $=0.0143$ ), which reduced significantly at a $95 \%$ $\mathrm{AuV}$ share (average $=0.0019$, standard deviation $=0.0014$ ). At $5 \% \mathrm{AuV}$ share, the average reduction in the CRI from the base case (average CRI $=0.0218$, standard deviation $=0.0149$ ) was $4.82 \%$ that gradually increased to a 91.35\% reduction at $95 \% \mathrm{AuV}$ share. For specific $\mathrm{AuV}$ shares, the CRIs experienced an upward trend with increasing inflow rates. Findings from this analysis conform to the findings of Papadoulis et al. [43], Ye and Yamamoto [11], and Rahman and Abdel-Aty [79], who all concluded, based on different sets of parameter analysis, that increasing $\mathrm{AuV}$ share could greatly improve traffic safety conditions.

While both analyses discussed so far showed significant improvements in overall traffic safety with a gradual increase of $\mathrm{AuV}$ shares, the findings were incomprehensible when determining the implications of AuVs on HuVs' perceived safety. Since the movements of AuVs were regarded as safe, irrespective of TTC values, due to their instantaneous response capability, an overall reduction of potential conflict events and crash risk for all traffic was anticipated with increasing $\mathrm{AuV}$ shares. Hence, adjusted CRI values were measured by considering only the number of $\mathrm{HuVs}$ passing through the weaving section to obtain more profound insights. The analysis results are presented in Figure 6(c). As portrayed in this figure, the CRI from the human drivers' perspective did not present as straightforward improvements as in the previous analysis. While the pattern of higher CRI with increasing inflow rate persisted for a specific $\mathrm{AuV}$ share, the reduction of CRI with increasing AuV share was renounced. The minimum average $\mathrm{CRI}$ for $\mathrm{HuVs}$ was experienced at $70 \% \mathrm{AuV}$ share $(0.0083)$ with minor changes until this point. However, the HuVs encountered substantially higher safety issues at high inflow rates from $75 \% \mathrm{AuV}$ share upwards (at 5\% AuV share). The maximum CRI at 95\% AUV share was 0.0966 that occurred at a $2800 \mathrm{vphpl}$ inflow rate, more than two times higher than the maximum CRI (0.0401) at a 5\% AuV rate. While the overall reduction 


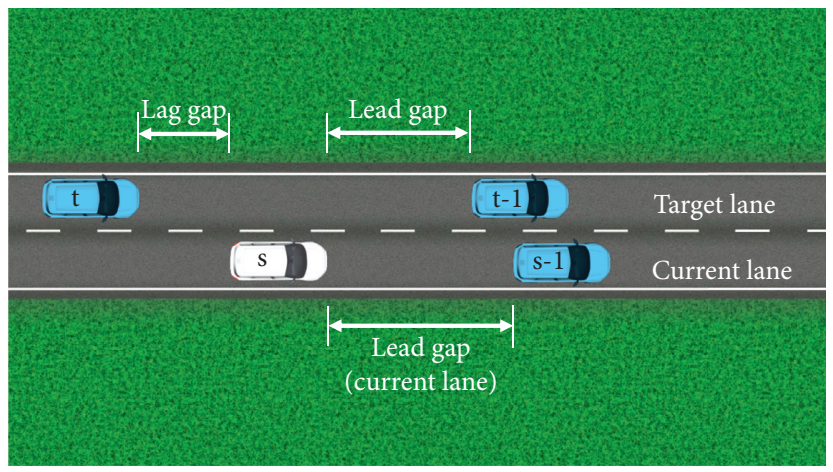

(a)

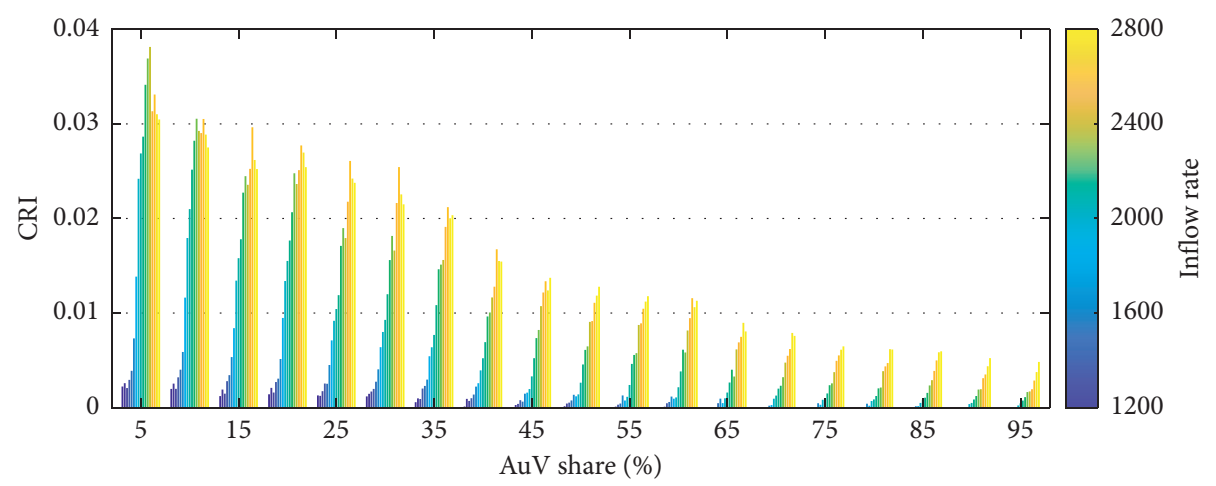

(b)

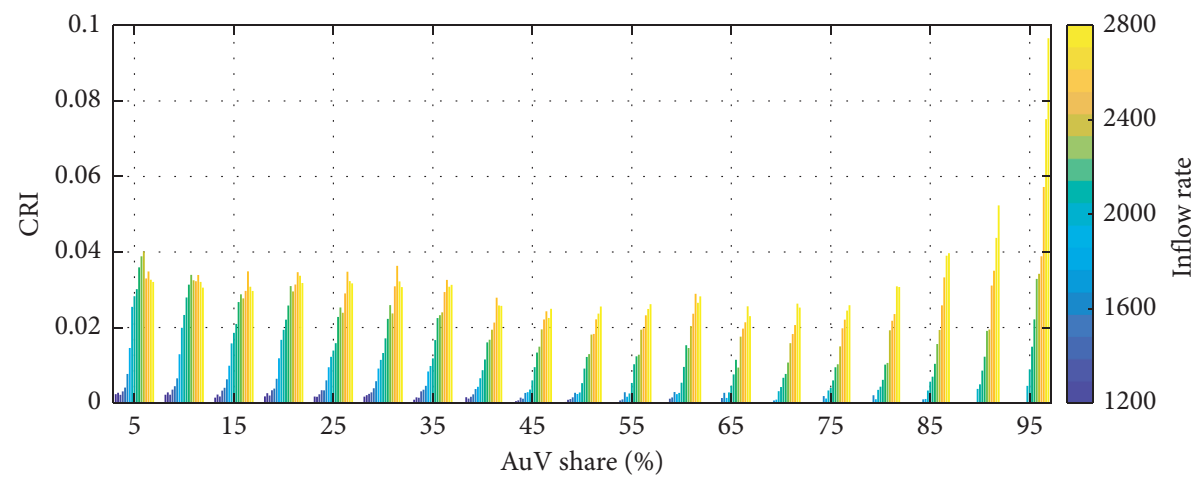

(c)

FIGURE 6: (a) Subject vehicle's perspective of decision parameters, and CRI values for simulated traffic states considering (b) all vehicles and (c) HuVs only.

in CRI up to the $75 \% \mathrm{AuV}$ share was moderate, the increments in CRI for HuVs were more drastic at high inflow rates with higher AuV shares.

6.2. Rear-End Crash Potential. Time Exposed Time-to-collision (TET) and Time Integrated Time-to-collision (TIT) are derived from TTC to perform as surrogate safety measures for vehicles at risk for rear-end crashes. Hayward et al. [80, 81] introduced TET and TIT, which have been widely used in the traffic safety literature to evaluate rear-end crash potential in traffic scenarios. TET is the summation of instances where the TTC is lower than a threshold value. In the previous section, the conflict events were identified similarly. However, the difference between the identified rear-end conflict events and TET measurements for rear-end crash potential stems from the fact that multiple consecutive TTC values for the subject vehicles lower than threshold TTC were counted as a single potential in the conflict event identification process.

On the other hand, individual timesteps are considered in the TET calculation. Therefore, higher TET values could be observed with lower rear-end conflict events, which would indicate higher safety concerns. TIT measures the value of TTC lower than threshold TTC. Similar to TET, lower TIT values are expected in safer traffic conditions. The values of these parameters were measured using the following equations: 


$$
\begin{aligned}
& \mathrm{TET}_{i}=\sum_{s=1}^{S} \mathrm{TET}_{s}, \\
& \mathrm{TET}_{s}=\sum_{k=1}^{K} \delta_{k} \Delta k, \\
& \delta_{k}= \begin{cases}1, & \forall 0<\mathrm{TTC}_{s}(k)<\mathrm{TTC}^{*}, \\
0, & \text { else, }\end{cases} \\
& \mathrm{TIT}_{i}=\sum_{s=1}^{S} \mathrm{TIT}_{s}, \\
& \mathrm{TIT}_{s}=\sum_{k=1}^{K}\left[\frac{1}{\mathrm{TTC}_{s}(k)}-\frac{1}{\mathrm{TTC}^{*}}\right] \cdot \Delta k, \\
& \forall 0<\mathrm{TTC}_{s}(k)<\mathrm{TTC}^{*} .
\end{aligned}
$$

Here, TET $_{i}$ and TIT $_{i}$ are the TET and TIT values for traffic scenario (i.e., inflow rate and $\mathrm{AuV}$ share) $i, \mathrm{TET}_{s}$ and $\mathrm{TIT}_{s}$ are the TIT and TIT values for subject vehicle $s, \mathrm{TTC}^{*}$ is threshold TTC (1.5 sec according to [77]), and $\mathrm{TTC}_{s}(k)$ is TTC of subject vehicle $s$ at timestep $k$. Aligned with the previous safety analysis, this analysis measured the TET and TIT values of simulated traffic for all 323 studied scenarios. The simulation instance with maximum throughput was considered for each scenario. Both TET and TIT counted for mixed traffic volume over the duration and adjusted for changing $\mathrm{HuV}$ proportions of traffic to make a fair comparison. As observed in Figures $7(\mathrm{a})$ and $7(\mathrm{c})$, both TET and TIT of mixed traffic gradually decreased with an increasing share of AuVs. Generally, for a specific AuV share, TET and TIT for mixed traffic increased with increasing inflow rates. This pattern for specific $\mathrm{AuV}$ shares persisted through adjusted TET and TIT. Figures 7(b) and 7(d) account for gradually diminishing shares of HuVs in traffic. Nevertheless, adjusted TET and TIT that had changed due to increasing AuV share were dissimilar to those for the mixed traffic scenario. Although the TET and TIT were reduced considerably from mixed traffic volume, these parameter values increased once considered from only $\mathrm{HuVs}$, particularly at high AuV shares.

This analysis demonstrated the dichotomy of crash potential due to changes in perspective. In plain sight, both TET and TIT showed significant reductions (from average TET $=100$ and $\mathrm{TIT}=0.1318$ at $5 \% \mathrm{AuV}$ share to average $\mathrm{TET}=3.3$ and $\mathrm{TIT}=0.0124$ at $95 \% \mathrm{AuV}$ share) for the mixed traffic passing through the weaving section. However, in-depth observations of these parameters from the $\mathrm{HuVs}$ ' perspective revealed stark dissimilarities at higher $\mathrm{AuV}$ shares (from average adjusted TET $=105$ and TIT $=0.1388$ at $5 \% \mathrm{AuV}$ share to average adjusted $\mathrm{TET}=65.88$ and $\mathrm{TIT}=0.2472$ at $95 \% \mathrm{AuV}$ share). It is also important to note that rear-end crash potential was nonexistent for the majority $(1200-2100 \mathrm{vphpl})$ of the simulated inflow rate. Hence, the increasing trend in rear-end crash risk by $\mathrm{HuVs}$ was pushed by extensive $\mathrm{AuV}$ presence at high inflow rates. Moreover, the analysis disclosed the ineffectiveness of AuVs to influence the perceived safety of $\mathrm{HuVs}$ at high inflow rates in weaving sections, even with leading market share.

\section{Maximizing Collective Mobility and Safety Implications}

Due to the complex correlation of simulated traffic scenarios (i.e., inflow rates and $\mathrm{AuV}$ shares) with mobility and safety implications, developing a closed-form objective function to attain combined optimal benefits is considerably complex and time-consuming. Hence, an alternative approach of iterative search with metamodeling was taken in this study. Metamodeling is a macromodeling method used in the literature [82-85] that aims to build simple and computationally inexpensive models, replicating the observed correlations when samples of a complex, high-fidelity model or simulation are drawn. Metamodels are often referred to as approximation, surrogate, and response surface models. Different metamodeling techniques include generating analytically inexpensive approximations of computationally intensive true response through various machine learning methods such as polynomial interpolation, support vector regression, kriging, and neural network [86-89]. This approach has been commonly used for solving simulationbased optimization and analytical dynamic equilibrium problems in transportation applications. The goal of including this modeling in this research is to produce a faster and simpler approximation of simulation-generated results to achieve multiobjective optimization and design space exploration feasible.

Mobility and safety scores were calculated for the range of simulated traffic scenarios from the attained parameter values of chosen mobility and safety parameters. To achieve balanced mobility benefits, both the average travel time and average travel distance were leveraged to calculate mobility scores. Safety scores were achieved by measuring the reduction of CRI from the base case. The following equations were used to measure the mobility and safety scores of each inflow rate and $\mathrm{AuV}$ share scenario:

$$
\begin{aligned}
\operatorname{MS}_{(i, j)} & =\sum_{t=1}^{T}\left[\rho_{(i, j)}(t)\left[v_{\mathrm{SL}}-v_{i, j}(t)\right]-\rho_{(i, 0)}(t)\left[v_{\mathrm{SL}}-v_{(i, 0)}(t)\right]\right], \\
\mathrm{SS}_{(i, j)} & =\mathrm{CRI}_{(i, 0)}-\mathrm{CRI}_{(i, j)} .
\end{aligned}
$$

Here, $\mathrm{MS}_{(i, j)}$ and $\mathrm{SS}_{(i, j)}$ are mobility and safety scores for inflow rate $I$ and $\mathrm{AuV}$ share $j ; \rho_{(i, j)}(t)$ and $v_{i, j}(t)$ are the traffic density and average traffic speed at the weaving section at timestep $t$ for inflow rate I and AuV share $j ; \rho_{(i, 0)}$ and $v_{(i, 0)}$ are the traffic density and average traffic speed at the base case $\left(0 \% \mathrm{AuV}\right.$ share); $v_{\mathrm{SL}}$ is the speed limit of the weaving section. For a better performance by the machine learning algorithm, both scores were scaled within a 0 to 1 range, as they would be supplied as training and testing samples of a neural network (Figures 8(a) and 8(b)). The neural network was developed in MATLAB with inflow rates and $\mathrm{AuV}$ shares as input and 


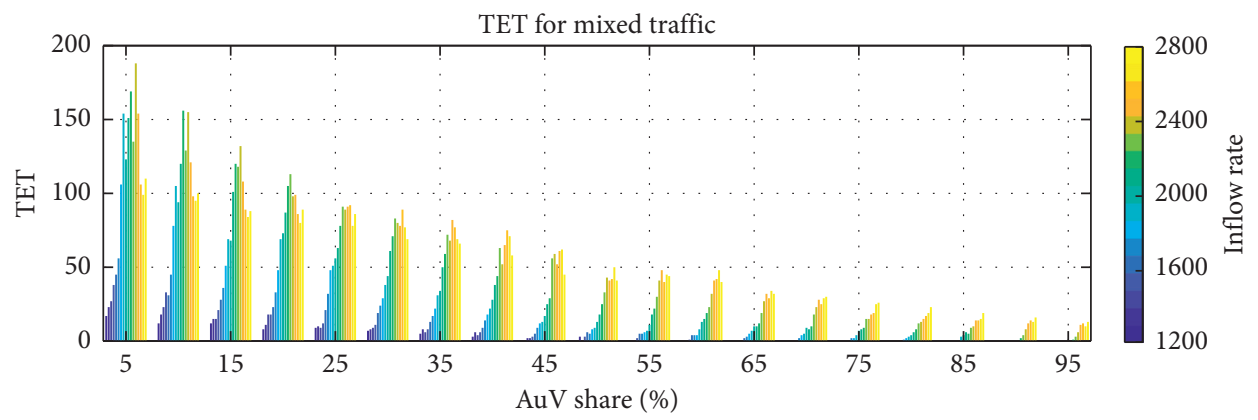

(a)

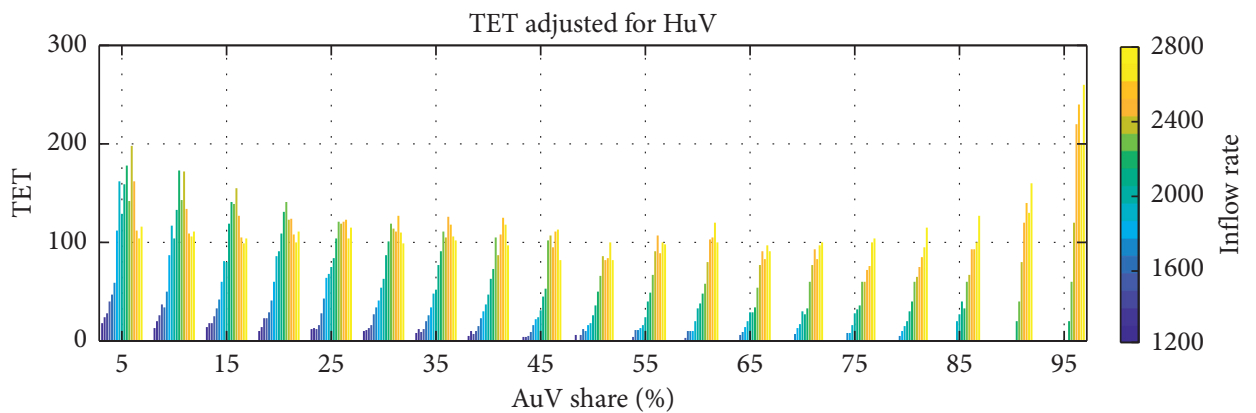

(b)

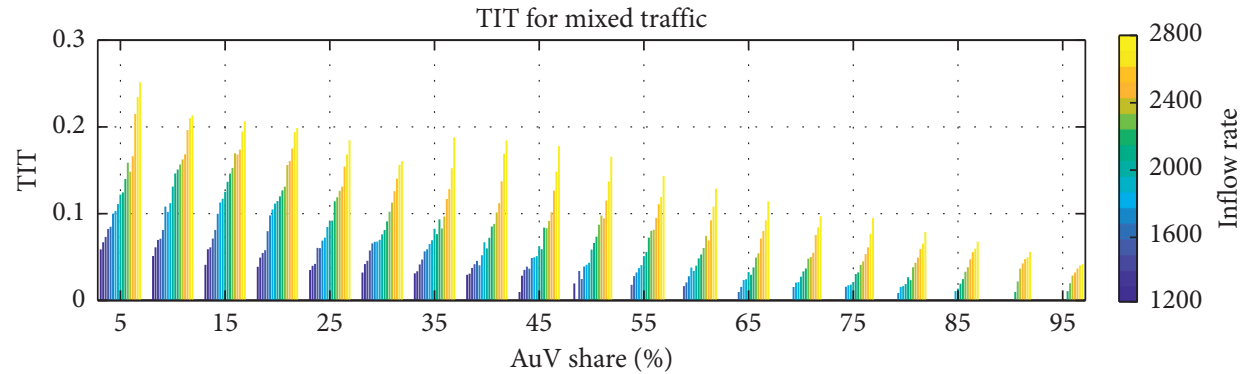

(c)

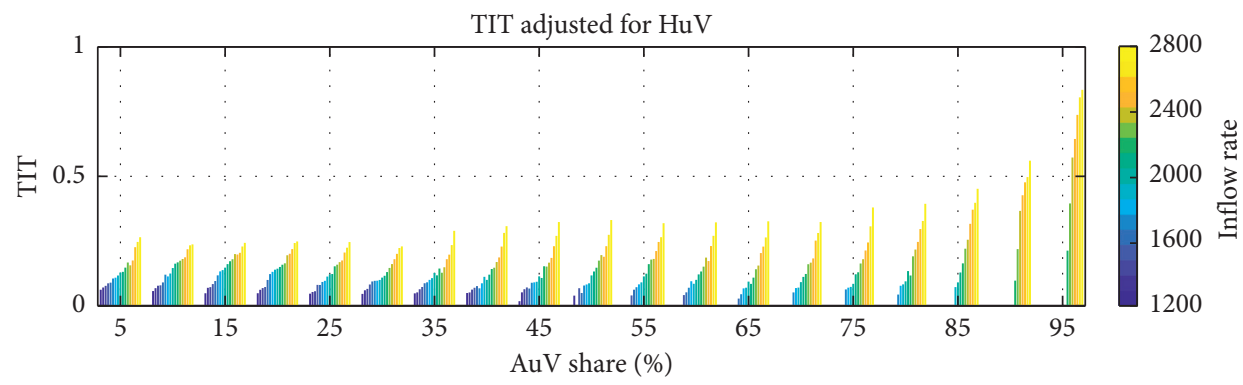

(d)

Figure 7: TET and TIT values in different traffic states for (a, c) total traffic and (b, d) HuVs only.

corresponding mobility and safety scores as output. Seventy percent of samples were used for training, and the remaining samples were used for testing purposes. A Bayesian regularization algorithm was chosen with 20 hidden layers. The performance of each iteration was measured by mean squared error. A final model comparison between predicted and expected values showed $R^{2}=0.9884$ for training samples and $R^{2}=0.9786$ for testing samples. The purpose of developing such metamodels was to regress the response surface that characterizes the correlation between decision variable inputs (i.e., inflow rates and $\mathrm{AuV}$ shares) and simulation outputs (i.e., mobility and safety score).

The finalized training model was then applied to obtain scaled mobility and safety scores for specific inflow rates and $\mathrm{AuV}$ shares in the weaving section. Furthermore, the model imparted optimal AuV share information to achieve maximum collective mobility and safety benefits for the specified inflow rate. Suppose the upstream average flow rate of the weaving section $1760 \mathrm{vphpl}$ and AuV share of the traffic is $46 \%$. The model provided the expected mobility and safety scores, which 


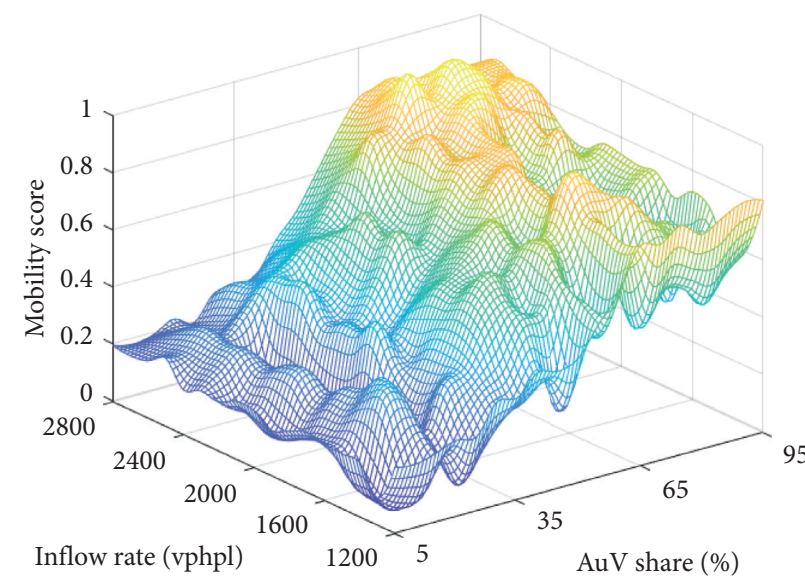

(a)

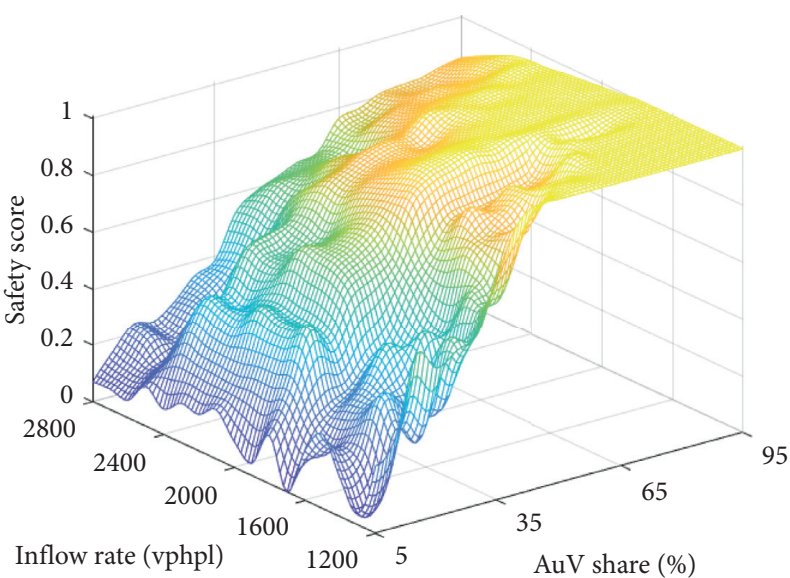

(b)

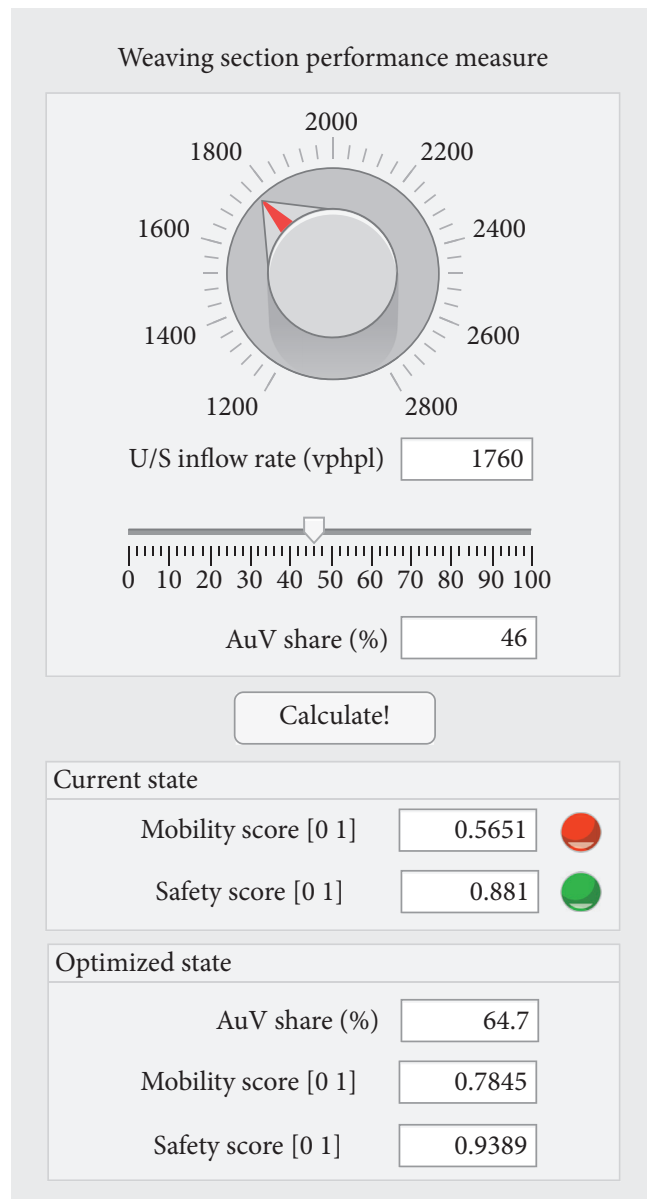

(c)

Figure 8: (a) Mobility scores, (b) safety scores for varying inflow rates and mixed traffic scenarios, and (c) user interface of the developed application to assist decision-making for weaving section performance.

were $\mathrm{MS}_{(1760,0.46)}=0.5651$ and $\mathrm{SS}_{(1760,0.46)}=0.8810$, respectively. For the same upstream average flow rate, an AuV share of $64.7 \%\left(\mathrm{MS}_{(1760,0.647)}=0.7845, \mathrm{SS}_{(1760,0.647)}=0.9389\right)$ would maximize the collective mobility and safety impact (Figure $8(\mathrm{c})$ ). To achieve more efficient flow and safer traffic movements through weaving sections that may act as bottlenecks on the freeway network, this information would be crucial for traffic operation and management authorities to impose controls enabling inflow rates and $\mathrm{AuV}$ share.

\section{Conclusion}

This paper proposed a microscopic modeling framework of mixed traffic that was applied to a freeway weaving section. 
Multifaceted traffic scenarios of mixed traffic were simulated for the weaving section, a notably underexplored combination in the literature. The modeling framework has been built on MPC-based decision-making principles that account for the anticipative aspects of human drivers. This modeling capability can be attributed to lateral control decisions and control model transitions of both vehicle types. Specifically, the cornerstone of reproducing realistic traffic states for a multilane roadway was anticipatory lanechanging decisions while perceiving the future status of neighboring vehicles and reference lane-changing trajectories built upon empirical observations. In this study, the simulation results contributed to understanding the effects of AuVs on traffic mobility and safety as well as validating the findings from previous relevant studies, such as Tilg et al. [34], Liu et al. [68], Spiliopoulou et al. [76], Papadoulis et al. [43], Ye and Yamamoto [11], and Rahman and Abdel-Aty [79]. While our findings were consistent with this research, our study contributes to the body of knowledge by addressing several underrepresented issues, including dichotomic interaction between mobility and safety in mixed traffic, pseudosafety perception with higher $\mathrm{AuV}$ shares, and metamodeling to attain equitable mobility and safety advantage from $\mathrm{AuV}$ presence in a weaving section.

Analysis results revealed that a low $\mathrm{AuV}$ share with high inflow rates could undermine the mobility of the weaving section. This finding was critical for understanding the place of the weaving section in these traffic scenarios due to the distinct and preemptive lane-changing activity experienced. Safety features of traffic in weaving sections were found to be exponentially associated with $\mathrm{AuV}$ shares. While increasing traffic demand could raise safety concerns at any level of $\mathrm{AuV}$ presence, the range of possible conflict levels was substantially reduced with higher $\mathrm{AuV}$ shares. Finally, the simulation results were assembled to develop an assistive application for traffic operation and management authorities, aiding traffic state evaluation from both mobility and safety perspectives. The application can also seek optimal levels of $\mathrm{AuV}$ presence to maximize the impact. Altogether, the analysis results were reported to provide clear insight into the implications of $\mathrm{AuVs}$ on weaving section traffic mobility and safety and clarify the transformation of mixed traffic flow dynamics with the gradual adoption of AuVs under the current traffic system. One limitation of this study is the counterintuitive result of attaining maximum throughput at $65 \% \mathrm{AuV}$ share. While the simulation model validation containing $\mathrm{HuVs}$ showed adequate resemblance to real-world traffic conditions, mixed traffic conditions may substantially differ. The current version of the developed model could have limited success in replicating the complex interaction between AuVs and HuVs. Therefore, further improvements are required prior to policy decision-making and should be based on real-world corroboration of $\mathrm{AuV}$ and $\mathrm{HuV}$ interactions.

Future research includes further expansion of human driving behavior and the identification of resulting variations. Furthermore, we will continue the research by considering different platoon strategies and distinguishing the impact of different platoon structures and varying human behavior. While this study examined the mobility and safety implications for traffic containing two types of vehicles, the mixed traffic scenario will be enhanced to address $\mathrm{HuVs}$ with an advanced driver assistance system that improves driving efficiency without absolute reliance on automation.

\section{Data Availability}

The simulated data used to support the findings of this study are available from the corresponding author upon request.

\section{Disclosure}

The contents of this paper reflect the views of the authors who are responsible for the facts and the accuracy of the data presented herein. The contents do not necessarily reflect the official views or policies of NSERC, City of Edmonton, and Transport Canada.

\section{Conflicts of Interest}

The authors declare that there are no conflicts of interest regarding the publication of this paper.

\section{Acknowledgments}

The authors want to acknowledge the support from the Natural Sciences and Engineering Research Council (NSERC) of Canada, City of Edmonton, and Transport Canada to provide funding for this research. The authors also acknowledge the contributions of Dr. Sharon Harper for her assistance in editing and proofreading the paper.

\section{References}

[1] H. He and M. Menendez, "WEAVE: capacity and level of service for freeway weaving areas," in Proceedings of the 2017 VSS Project BK Meeting, Zurich, Switzerland, 2017.

[2] F. Marczak, W. Daamen, and C. Buisson, "Empirical analysis of lane changing behavior at a freeway weaving section," in Proceedings of the 93rd Annual Meeting of the Transportation Research Board, Washington, DC, USA, 2014.

[3] J. H. Lee, Observations on Traffic Behavior in Freeway Weaving Bottlenecks: Empirical Study and Theoretical Modeling, University of California, Berkeley, CA, USA, 2008.

[4] H. He and M. Menendez, "Distribution and impacts of lane changes at a freeway weaving section: an empirical study," in Proceedings of the 95th Annual Meeting of the Transportation Research Board, Washington, DC, USA, 2016.

[5] T. F. Golob, W. W. Recker, and V. M. Alvarez, "Safety aspects of freeway weaving sections," Transportation Research Part A: Policy and Practice, vol. 38, no. 1, pp. 35-51, 2004.

[6] J. Fazio, J. Holden, and N. M. Rouphail, "Use of freeway conflict rates as an alternative to crash rates in weaving section safety analyses," Transportation Research Record, vol. 1401, pp. 61-69, 1993.

[7] N. Uno, Y. Iida, S. Itsubo, and S. Yasuhara, "A microscopic analysis of traffic conflict caused by lane-changing vehicle at weaving section," in Proceedings of the 13th Mini-EURO Conference on Handling Uncertainty in the Analysis of Traffic and Transportation Systems, pp. 143-148, Bari, Italy, 2003. 
[8] S. S. Pulugurtha and J. Bhatt, "Evaluating the role of weaving section characteristics and traffic on crashes in weaving areas," Traffic Injury Prevention, vol. 11, no. 1, pp. 104-113, 2010.

[9] A. Ghiasi, O. Hussain, Z. Qian, and X. Li, "A mixed traffic capacity analysis and lane management model for connected automated vehicles: a Markov chain method," Transportation Research Part B: Methodological, vol. 106, pp. 266-292, 2017.

[10] M. Seraj, J. Li, and T. Z. Qiu, "Modeling microscopic carfollowing strategy of mixed traffic to identify optimal platoon configurations for multiobjective decision-making," Journal of Advanced Transportation, vol. 2018, Article ID 7835010, 15 pages, 2018.

[11] L. Ye and T. Yamamoto, "Evaluating the impact of connected and autonomous vehicles on traffic safety," Physica A: Statistical Mechanics and Its Applications, vol. 526, Article ID 121009, 2019.

[12] W. X. Zhu and H. M. Zhang, "Analysis of mixed traffic flow with human-driving and autonomous cars based on carfollowing model," Physica A: Statistical Mechanics and Its Applications, vol. 496, pp. 274-285, 2018.

[13] K. Götz, "Traffic mobility," in Encyclopedia of Quality of Life and Well-Being Research, pp. 6705-6710, Springer, Dordrecht, Netherlands, 2014.

[14] P. Liu, U. Ozguner, and Y. Zhang, "Distributed MPC for cooperative highway driving and energy-economy validation via microscopic simulations," Transportation Research Part C: Emerging Technologies, vol. 77, pp. 80-95, 2017.

[15] L. Bertoni, J. Guanetti, M. Basso, M. Masoero, S. Cetinkunt, and F. Borrelli, "An adaptive cruise control for connected energy-saving electric vehicles," IFAC-PapersOnLine, vol. 50, no. 1, pp. 2359-2364, 2017.

[16] T. Stanger and L. del Re, "A model predictive cooperative adaptive cruise control approach," in Proceedings of the 2013 American Control Conference, pp. 1374-1379, Washington, DC, USA, 2013.

[17] M. A. S. Kamal, S. Taguchi, and T. Yoshimura, "Efficient driving on multilane roads under a connected vehicle environment," IEEE Transactions on Intelligent Transportation Systems, vol. 17, no. 9, pp. 2541-2551, 2016.

[18] S. Shladover, J. VanderWerf, M. A. Miller, N. Kourjanskaia, and H. Krishnan, Development and Performance Evaluation of AVCSS Deployment Sequences to Advance from Today's Driving Environment to Full Automation, UC Berkeley, Berkeley, CA, USA, 2001.

[19] J. Lee, J. Bared, and B. Park, "Mobility impacts of cooperative adaptive cruise control (CACC) under mixed traffic conditions," in Proceedings of the 21st World Congress on Intelligent Transport Systems: Reinventing Transportation in Our Connected World, Detroit, MI, USA, 2014.

[20] J. Vander Werf, S. Shladover, M. Miller, and N. Kourjanskaia, "Effects of adaptive cruise control systems on highway traffic flow capacity," Transportation Research Record: Journal of the Transportation Research Board, vol. 1800, no. 2, pp. 78-84, 2002.

[21] B. Van Arem, C. J. G. Van Driel, and R. Visser, "The impact of cooperative adaptive cruise control on traffic-flow characteristics," IEEE Transactions on Intelligent Transportation Systems, vol. 7, no. 4, pp. 429-436, 2006.

[22] A. Kesting, M. Treiber, M. Schönhof, and D. Helbing, "Adaptive cruise control design for active congestion avoidance," Transportation Research Part C: Emerging Technologies, vol. 16, no. 6, pp. 668-683, 2008.

[23] A. Kesting, M. Treiber, and D. Helbing, "Enhanced intelligent driver model to access the impact of driving strategies on traffic capacity," Philosophical Transactions of the Royal Society A: Mathematical, Physical and Engineering Sciences, vol. 368, no. 1928, pp. 4585-4605, 2010.

[24] S. E. Shladover, D. Su, and X. Y. Lu, "Impacts of cooperative adaptive cruise control on freeway traffic flow," Transportation Research Record, vol. 2324, pp. 63-70, 2012.

[25] P. Tientrakool, Y.-C. Ho, and N. F. Maxemchuk, "Highway capacity benefits from using vehicle-to-vehicle communication and sensors for collision avoidance," in Proceedings of the 2011 IEEE Vehicular Technology Conference (VTC Fall), pp. 1-5, San Francisco, CA, USA, 2011.

[26] D. Chen, S. Ahn, M. Chitturi, and D. A. Noyce, "Towards vehicle automation: roadway capacity formulation for traffic mixed with regular and automated vehicles," Transportation Research, Series B: Methodological, vol. 100, pp. 196-221, 2017.

[27] H. Liu, X. Kan, S. E. Shladover, X. Y. Lu, and R. A. Ferlis, "Quantifying Influences of cooperative adaptive cruise control (CACC) vehicle string operation strategies on mixed traffic flow," in Proceedings of the Transportation Research Board 97th Annual Meeting, Washington, DC, USA, 2018.

[28] R. Fu, Z. Li, Q. Sun, and C. Wang, "Human-like car-following model for autonomous vehicles considering the cut-in behavior of other vehicles in mixed traffic," Accident Analysis \& Prevention, vol. 132, Article ID 105260, 2019.

[29] B. Khondaker and L. Kattan, "Variable speed limit: a microscopic analysis in a connected vehicle environment," Transportation Research Part C: Emerging Technologies, vol. 58, pp. 146-159, 2015.

[30] S. Hong, A. A. Malikopoulos, B. B. Park, and J. Lee, "Speed harmonization using optimal control algorithm under mixed traffic of connected-automated and human driven vehicles," in Proceedings of the 2018 Transportation Research Board Annual Meeting, Washington, DC, USA, 2018.

[31] Y. Han, D. Chen, and S. Ahn, "Variable speed limit control at fixed freeway bottlenecks using connected vehicles," Transportation Research Part B: Methodological, vol. 98, pp. 113134, 2017.

[32] A. A. Malikopoulos, S. Hong, B. B. Park, J. Lee, and S. Ryu, "Optimal control for speed harmonization of automated vehicles," IEEE Transactions on Intelligent Transportation Systems, vol. 20, no. 7, pp. 2405-2417, 2019.

[33] J. Rios-Torres and A. A. Malikopoulos, "Impact of partial penetrations of connected and automated vehicles on fuel consumption and traffic flow," IEEE Transactions on Intelligent Vehicles, vol. 3, no. 4, pp. 453-462, 2018.

[34] G. Tilg, K. Yang, and M. Menendez, "Evaluating the effects of automated vehicle technology on the capacity of freeway weaving sections," Transportation Research Part C: Emerging Technologies, vol. 96, pp. 3-21, 2018.

[35] L. Ye and T. Yamamoto, "Modeling connected and autonomous vehicles in heterogeneous traffic flow," Physica A: Statistical Mechanics and Its Applications, vol. 490, pp. 269277, 2018.

[36] L. Ye and T. Yamamoto, "Impact of dedicated lanes for connected and autonomous vehicle on traffic flow throughput," Physica A: Statistical Mechanics and Its Applications, vol. 512, pp. 588-597, 2018.

[37] B. Hayes, "Leave the driving to your car?" IEEE Spectrum, vol. 39, no. 4, p. 9, 2002.

[38] D. J. Fagnant and K. Kockelman, "Preparing a nation for autonomous vehicles: opportunities, barriers and policy recommendations," Transportation Research Part A: Policy and Practice, vol. 77, pp. 167-181, 2015. 
[39] R. Fan, H. Yu, P. Liu, and W. Wang, "Using VISSIM simulation model and surrogate safety assessment model for estimating field measured traffic conflicts at freeway merge areas," IET Intelligent Transport Systems, vol. 7, no. 1, pp. 68-77, 2013.

[40] F. Huang, P. Liu, H. Yu, and W. Wang, "Identifying if VISSIM simulation model and SSAM provide reasonable estimates for field measured traffic conflicts at signalized intersections," Accident Analysis \& Prevention, vol. 50, pp. 1014-1024, 2013.

[41] M. Essa and T. Sayed, "Transferability of calibrated microsimulation model parameters for safety assessment using simulated conflicts," Accident Analysis \& Prevention, vol. 84, pp. 41-53, 2015.

[42] U. Shahdah, F. Saccomanno, and B. Persaud, "Application of traffic microsimulation for evaluating safety performance of urban signalized intersections," Transportation Research Part C: Emerging Technologies, vol. 60, pp. 96-104, 2015.

[43] A. Papadoulis, M. Quddus, and M. Imprialou, "Evaluating the safety impact of connected and autonomous vehicles on motorways," Accident Analysis \& Prevention, vol. 124, pp. 12-22, 2019.

[44] J. Zhang, K. Wu, M. Cheng, M. Yang, Y. Cheng, and S. Li, "Safety evaluation for connected and autonomous vehicles' exclusive lanes considering penetrate ratios and impact of trucks using surrogate safety measures," Journal of Advanced Transportation, vol. 2020, Article ID 5847814, 16 pages, 2020.

[45] S. Moon, I. Moon, and K. Yi, "Design, tuning, and evaluation of a full-range adaptive cruise control system with collision avoidance," Control Engineering Practice, vol. 17, no. 4, pp. 442-455, 2009.

[46] Y. Li, H. Wang, W. Wang, L. Xing, S. Liu, and X. Wei, "Evaluation of the impacts of cooperative adaptive cruise control on reducing rear-end collision risks on freeways," Accident Analysis \& Prevention, vol. 98, pp. 87-95, 2017.

[47] N. Virdi, H. Grzybowska, S. T. Waller, and V. Dixit, "A safety assessment of mixed fleets with connected and autonomous vehicles using the surrogate safety assessment module," Accident Analysis \& Prevention, vol. 131, pp. 95-111, 2019.

[48] Y. Tu, W. Wang, Y. Li, C. Xu, T. Xu, and X. Li, "Longitudinal safety impacts of cooperative adaptive cruise control vehicle's degradation," Journal of Safety Research, vol. 69, pp. 177-192, 2019.

[49] Y. Shi, Y. Li, Q. Cai, H. Zhang, and D. Wu, "How does heterogeneity affect freeway safety? A simulation-based exploration considering sustainable intelligent connected vehicles," Sustainability, vol. 12, no. 21, p. 8941, 2020.

[50] R. Arvin, A. J. Khattak, M. Kamrani, and J. Rio-Torres, "Safety evaluation of connected and automated vehicles in mixed traffic with conventional vehicles at intersections," Journal of Intelligent Transportation Systems, vol. 25, no. 2, pp. 1-18, 2020.

[51] N. Eissfeldt and P. Wagner, "Effects of anticipatory driving in a traffic flow model," European Physical Journal B: Condensed Matter and Complex Systems, vol. 33, no. 1, pp. 121-129, 2003.

[52] H. Lenz, C. K. Wagner, and R. Sollacher, "Multi-anticipative car-following model," European Physical Journal B: Condensed Matter and Complex Systems, vol. 7, no. 2, pp. 331-335, 1999.

[53] W. Knospe, L. Santen, A. Schadschneider, and M. Schreckenberg, "Human behavior as origin of traffic phases," Physical Review E, vol. 65, no. 1, Article ID 015101, 2001.

[54] H. K. Lee, R. Barlovic, M. Schreckenberg, and D. Kim, "Mechanical restriction versus human overreaction triggering congested traffic states," Physical Review Letters, vol. 92, no. 23, Article ID 238702, 2004.

[55] S. N. J. Watamaniuk and S. J. Heinen, "Perceptual and oculomotor evidence of limitations on processing accelerating motion," Journal of Vision, vol. 3, no. 11, p. 5, 2003.

[56] M. Treiber, A. Kesting, and D. Helbing, "Delays, inaccuracies and anticipation in microscopic traffic models," Physica A: Statistical Mechanics and Its Applications, vol. 360, no. 1, pp. 71-88, 2006.

[57] M. Lindorfer, C. F. Mecklenbrauker, and G. Ostermayer, "Modeling the imperfect driver: incorporating human factors in a microscopic traffic model," IEEE Transactions on Intelligent Transportation Systems, vol. 19, no. 9, pp. 2856-2870, 2018.

[58] M. Makridis, K. Mattas, D. Borio, R. Giuliani, and B. Ciuffo, "Estimating reaction time in adaptive cruise control system," in Proceedings of the 2018 IEEE Intelligent Vehicles Symposium (IV), Changshu, China, 2018.

[59] Q. Deng, “A general simulation framework for modeling and analysis of heavy-duty vehicle platooning," IEEE Transactions on Intelligent Transportation Systems, vol. 17, no. 11, pp. 3252-3262, 2016.

[60] L. L. Hoberock, A Survey of Longitudinal Acceleration Comfort Studies in Ground Transportation Vehicles, Council for Advanced Transportation Studies, University of Texas at Austin, Austin, TX, USA, 1976.

[61] M. Treiber, A. Hennecke, and D. Helbing, "Congested traffic states in empirical observations and microscopic simulations," Physical Review E, vol. 62, no. 2, pp. 1805-1824, 2000.

[62] S. G. Hu, H. Y. Wen, L. Xu, and H. Fu, "Stability of platoon of adaptive cruise control vehicles with time delay," Transportation Letters, vol. 7867, pp. 1-10, 2017.

[63] D. Bezzina and J. Sayer, Safety Pilot Model Deployment: Test Conductor Team Report, National Highway Traffic Safety Administration, Washington, DC, USA, 2015.

[64] L. Tijerina, W. R. Garrott, D. Stoltzfus, and E. Parmer, "Eye glance behavior of van and passenger car drivers during lane change decision phase," Transportation Research Record: Journal of the Transportation Research Board, vol. 1937, no. 1, pp. 37-43, 2005.

[65] G. Lee, Modeling Gap Acceptance at Freeway Merges, Massachusetts Institute of Technology, Cambridge, MA, USA, 2006.

[66] T. Toledo and D. Zohar, "Modeling duration of lane changes," Transportation Research Record: Journal of the Transportation Research Board, vol. 1999, no. 1, pp. 71-78, 2007.

[67] C. Thiemann, M. Treiber, and A. Kesting, "Estimating acceleration and lane-changing dynamics from next generation simulation trajectory data," Transportation Research Record: Journal of the Transportation Research Board, vol. 2088, no. 1, pp. 90-101, 2008.

[68] H. Liu, X. Kan, S. E. Shladover, X. Y. Lu, and R. E. Ferlis, "Modeling impacts of cooperative adaptive cruise control on mixed traffic flow in multilane freeway facilities," Transportation Research Part C: Emerging Technologies, vol. 95, pp. 261-279, 2018.

[69] T. H. Chang and I. S. Lai, "Analysis of characteristics of mixed traffic flow of autopilot vehicles and manual vehicles," Transportation Research Part C: Emerging Technologies, vol. 5, no. 6, pp. 333-348, 1997.

[70] P. Fernandes and U. Nunes, "Platooning with IVC-enabled autonomous vehicles: strategies to mitigate communication delays, improve safety and traffic flow," IEEE Transactions on 
Intelligent Transportation Systems, vol. 13, no. 1, pp. 91-106, 2012.

[71] D. Ni, J. Li, S. Andrews, and H. Wang, "Preliminary estimate of highway capacity benefit attainable with IntelliDrive technologies," in Proceedings of the 13th International IEEE Conference on Intelligent Transportation Systems, pp. 819-824, Funchal, Portugal, 2010.

[72] V. A. C. van den Berg and E. T. Verhoef, "Autonomous cars and dynamic bottleneck congestion: the effects on capacity, value of time and preference heterogeneity," Transportation Research Part B: Methodological, vol. 94, pp. 43-60, 2016.

[73] A. Rezaei and B. Caulfield, "Simulating a transition to autonomous mobility," Simulation Modelling Practice and Theory, vol. 106, Article ID 102175, 2021.

[74] R. Jiang, C.-J. Jin, H. M. Zhang et al., "Experimental and empirical investigations of traffic flow instability," Transportation Research Part C: Emerging Technologies, vol. 94, pp. 83-98, 2018.

[75] A. Talebpour and H. S. Mahmassani, "Influence of connected and autonomous vehicles on traffic flow stability and throughput," Transportation Research Part C: Emerging Technologies, vol. 71, pp. 143-163, 2016.

[76] A. Spiliopoulou, D. Manolis, F. Vandorou, and M. Papageorgiou, "Adaptive cruise control operation for improved motorway traffic flow," in Proceedings of the 97th Annual Meeting of the Transportation Research Board, vol. 2250, Washigngton, DC, USA, 2018.

[77] D. Gettman, L. Pu, T. Sayed, and S. Shelby, "Surrogate safety assessment model and validation," Final report, FHWA, US Department of Transportation, Washington, DC, USA, 2008.

[78] C. Oh and T. Kim, "Estimation of rear-end crash potential using vehicle trajectory data," Accident Analysis \& Prevention, vol. 42, no. 6, pp. 1888-1893, 2010.

[79] M. S. Rahman and M. Abdel-Aty, "Longitudinal safety evaluation of connected vehicles' platooning on expressways," Accident Analysis \& Prevention, vol. 117, pp. 381-391, 2017.

[80] J. C. Hayward, "Near-miss determination through use of a scale of danger," in Proceedings of the 51st Annual Meeting of the Highway Research Board, pp. 24-35, Washington, DC, USA, 1971.

[81] M. M. Minderhoud and P. H. L. Bovy, "Extended time-tocollision measures for road traffic safety assessment," Accident Analysis \& Prevention, vol. 33, no. 1, pp. 89-97, 2001.

[82] A. Forrester, A. Sobester, and A. Keane, Engineering Design via Surrogate Modelling: A Practical Guide, John Wiley \& Sons, Hoboken, NJ, USA, 2008.

[83] E. I. Vlahogianni, M. G. Karlaftis, and J. C. Golias, "Optimized and meta-optimized neural networks for short-term traffic flow prediction: a genetic approach," Transportation Research Part C: Emerging Technologies, vol. 13, no. 3, pp. 211-234, 2005.

[84] E. I. Vlahogianni, "Optimization of traffic forecasting: intelligent surrogate modeling," Transportation Research Part C: Emerging Technologies, vol. 55, pp. 14-23, 2015.

[85] V. Boschian, M. Dotoli, M. P. Fanti, G. Iacobellis, and W. Ukovich, "A metamodeling approach to the management of intermodal transportation networks," IEEE Transactions on Automation Science and Engineering, vol. 8, no. 3, pp. 457469, 2011.

[86] A. Gosavi, Simulation-Based Optimization, Springer, Berlin, Germany, 2015.

[87] M. F. Hussain, R. R. Barton, and S. B. Joshi, "Metamodeling: radial basis functions, versus polynomials," European Journal of Operational Research, vol. 138, no. 1, pp. 142-154, 2002.
[88] S. Jakobsson, M. Patriksson, J. Rudholm, and A. Wojciechowski, "A method for simulation based optimization using radial basis functions," Optimization and Engineering, vol. 11, no. 4, pp. 501-532, 2010.

[89] N. V. Queipo, R. T. Haftka, W. Shyy, T. Goel, R. Vaidyanathan, and P. Kevin Tucker, "Surrogate-based analysis and optimization," Progress in Aerospace Sciences, vol. 41, no. 1, pp. 1-28, 2005. 